\title{
Introducing tools to test Higgs boson interactions via $W W$ scattering: One-loop calculations and renormalization in the Higgs effective field theory
}

\author{
Iñigo Asiáin $\odot^{*}$ Domènec Espriu $\odot,{ }^{\dagger}$ and Federico Mescia $\odot^{*}$ \\ Departament de Física Quàntica i Astrofísica, Institut de Ciències del Cosmos (ICCUB), \\ Universitat de Barcelona, Martí Franquès 1, 08028 Barcelona, Spain
}

(Received 15 September 2021; accepted 13 December 2021; published 7 January 2022)

\begin{abstract}
Effective field theories are useful tools to search for physics beyond the Standard Model (SM). However, effective theories can lead to nonunitary behavior with fast growing amplitudes. This unphysical behavior may induce a too large sensitivity to SM deviations, making necessary a unitarization of the amplitudes prior to a comparison with experiment. In the present work, we focus on all the processes entering twoHiggs production via longitudinal $W W$ scattering. We perform a one-loop calculation in the Higgs effective field theory framework of all relevant processes, determining the necessary counterterms in the on-shell scheme, and we study how the full inclusion of the gauge degrees of freedom modifies the previously computed masses and widths of the dynamical resonances arising from the unitarization process in the vector-isovector channel. Altogether, we are able to provide the technical tools that are needed to study the low-energy couplings in the Higgs effective theory under the requirements of unitarity and causality.
\end{abstract}

DOI: 10.1103/PhysRevD.105.015009

\section{INTRODUCTION}

Since the discovery in 2012 of a light scalar by ATLAS [1] and CMS [2], so far compatible with the Standard Model (SM) Higgs, a lot of questions have arisen regarding the origin of such a scalar and hence the properties of the electroweak symmetry breaking sector (EWSBS) [3-9].

To explore the nature of EWSBS beyond the SM (BSM), the scattering of longitudinally polarized electroweak gauge bosons is one of the most sensitive channels. The appearance of heavy resonances in the scattering of longitudinally polarized gauge bosons, for example, will be a clear indication of the existence of a strong dynamics behind EWSB [10-14].

The main properties of these resonances can be studied using effective theory treatment together with partial wave analysis and unitarization techniques [10,15-20]. Over the years, the use of inverse amplitude method (IAM) to build unitarity amplitudes has been successfully applied to explain the resonances in the pion-pion scattering [21-28]. The IAM allows us to predict mass and width of

\footnotetext{
*iasiain@icc.ub.edu †espriu@icc.ub.edu

mescia@ub.edu
}

Published by the American Physical Society under the terms of the Creative Commons Attribution 4.0 International license. Further distribution of this work must maintain attribution to the author(s) and the published article's title, journal citation, and DOI. Funded by SCOAP. dynamically generated resonances from the unitarized amplitudes of the low-energy effective theory. In turn, this also allow us to set bounds on the couplings of the underlying effective theories.

Among the open questions of EWSBS is the nature of the Higgs potential. Even if one assumes that the Higgs-like scalar found is truly elementary, are its self-interactions the ones predicted by the textbook SM?

Indeed, this is one of the main purposes of future machines such as the future ILC linear collider in Japan or the planned FCC $e^{+} e^{-}$at CERN [29]. In all these cases, setting bounds on effective couplings needs a bona fide and fair comparison that requires using unitarized amplitudes when departures from the SM values could be potentially large. The reason is that deviations of the couplings in the effective theory from their SM values lead to rapidly increasing cross sections and this may artificially enhance the sensitivity to the said couplings.

The purpose of this article is to provide some tools that would make this comparison possible. More specifically, we compute the renormalization counterterms at one loop that are required to calculate the processes $W_{L} W_{L} \rightarrow W_{L} W_{L}$, $W_{L} W_{L} \rightarrow h h$, and $h h \rightarrow h h$. All these amplitudes enter the unitarization of the $I, J=0,0$ channel. Moreover, we also provide for these $2 \rightarrow 2$ processes the corresponding renormalized amplitudes. Here, we calculate the full $\mathcal{O}\left(g^{2}\right)$ contributions of these processes. This paper thus completes and extends our previous work in Refs. $[10,15,16]$, where the analysis was carried out in the limit of no gauge interactions, $g=0$; only the longitudinal parts of the EW bosons, i.e., Goldstone bosons (GBs), were taken into 
account inside the loops. In the present work, as mentioned, we relax that approximation and allow transverse modes to propagate in the process, improving a weak point of the previous unitarization studies because along with the assumption $g=0$ in $[10,15,16]$, the authors consistently set $M_{W}=0$ for the real part of the loop calculation. With respect to previous works, we also compute the processes involving double Higgs production.

The derivation is made in the framework of the Higgs effective field theory (HEFT) [3-9,12], where the global symmetries are nonlinearly realized and the complex doublet structure of the SM is not assumed a priori (see e.g., the discussions in [30]). The calculation of the $2 \rightarrow 2$ physical amplitudes needed at the one-loop level beyond the SM is relatively involved. Thus, it is useful to take some shortcuts in order to have more manageable expressions. The real part of the amplitudes will be computed using the equivalence theorem [31-38] (ET), where the longitudinal components of $W$ in the external states are substituted by their Goldstone bosons (GBs). This approach is fully consistent in order to study cross-section of longitudinal polarized $W$ at energies much larger of the EW scale. The imaginary part of our amplitude is exactly obtained via the optical theorem, where physical $W$ are present in the external legs.

In this work, we make no assumption about the UV strong dynamics, In our model-independent study, the effects of the high-energy theory in the low energy regime are encoded in the so called chiral parameters. When these parameters do not have a correspondence in the SM, their presence spoils the unitarity of the amplitudes leading them, after unitarization, to exhibit resonances, i.e., bound states presumably resulting from the underlying strong dynamics.

For the purposes of this study, the custodial symmetry is assumed to remain exact and the soft breaking of the global symmetry $S U(2)_{L} \times S U(2)_{R}$ induced by the gauging of the hypercharge group will be neglected. We believe this approximation to be well justified by the experimental results of the $\rho$ parameter. In this limit, the electromagnetism is removed from the fundamental interactions and the gauge bosons transform exactly as a triplet under the vector (or custodial) subgroup after the global symmetry breaking pattern $S U(2)_{L} \times S U(2)_{R} \rightarrow S U(2)_{V}$, where $V$ stands for $L=R$. The absence of electromagnetic interactions moves the pole of $Z$ to the very same position of that of the $W$, making the $\rho$ parameter exactly equal to one at every order in perturbation theory.

As mentioned above, a full derivation of the one-loop counterterms in the HEFT, with a dynamical Higgs, is a necessary step in the process. A previous calculation of all the required counterterms does exist in the literature $[39,40]$. However, their expressions are not easily translated to the calculation of physical processes, we are interested in. For one thing, the renormalization scheme is not the widely-used on-shell scheme to which we adhere. On the other hand in $[39,40]$ extensive use is made of the equations of motion and field redefinitions, including some mixing of operators with different chiral dimensions. All this makes their results difficult or impossible to translate to a $S$-matrix calculation. Recently, an independent diagrammatic calculation was published [41], where a large set of counterterms are derived off shell (but only those needed for elastic vector boson scattering). We will review below our agreement with these preexisting results. It is worthwhile emphasizing that our approach is purely diagrammatic and inspired by the practical requirements needed when $S$-matrix elements are to be computed.

In the interest of practicality a number of simplifications have been made. They do not impact in any significant way the validity or relevance of the results. Let us list them here for the sake of clarity: (a) The equivalence theorem has been used to compute the real part of the one-loop correction. This does not restrict in any way the ability to obtain all the appropriate counterterms and it is an efficient way of arranging the calculation. This approximation also bypasses some subtleties related to crossing that will be pointed out below. (b) The equations of motion are systematically used; our results are relevant for on-shell processes and we actually have nothing to say for off-shell Green functions. Unlike the previous one, this approximation does reduce the number of contributing effective operators respect to the full list that is provided in the references [41,42], many of which are redundant when the equations of motion are used. (c) We work within the HEFT under the approximation of considering only custodially symmetric operators. This reduces even further the number of required operators and also implies the so-called isospin limit where $M_{W}=M_{Z}$. This approximation is, of course, numerically irrelevant for physics in the $\mathrm{TeV}$ region. But, in fact, there is a deeper reason that makes this approximation convenient; we set $g^{\prime}=0$ and neglect accordingly electromagnetism because it would not be possible to use the usual isospin decomposition otherwise and, in addition, long range interactions are not easily amenable to unitarization techniques. Obviously, electromagnetism should not be involved in any strong dynamics that may be present in the EWSBS. (d) Accordingly, custodially breaking operators are not included, as previously indicated; it would be inconsistent to include these and leave out the main source of weak isospin breaking in the SM. (e) The calculation is made in the Landau gauge, which simplifies somewhat the counterterm structure.

\section{THE EFFECTIVE LAGRANGIAN}

The electroweak chiral Lagrangian is a nonlinear gauged effective field theory mimicking chiral perturbation theory, used to investigate low-energy QCD, in the electroweak sector [43]. It has been used intensively in the context of 
effective field theories since the early days of LEP [43-45] in order to put to the test extensions of the SM. In the case of the electroweak sector, it only assumes the local and global properties known to hold at low energies, and makes no specific commitment to the underlying physics. The addition of the Higgs scalar makes this model a Higgs effective field theory.

This HEFT contains as dynamical fields, the EW gauge bosons $W^{ \pm}, Z, \gamma$; their associated Goldstone partners $\omega^{a}=\omega^{ \pm}, z$; and a light Higgs $h$ (the latter could or could not be a Goldstone boson). In the HEFT the Goldstones resulting from the electroweak breaking are described by a unitary matrix $U$ that takes values in the coset $S U(2)_{L} \times$ $S U(2)_{R} / S U(2)_{V}$ and the Higgs is a $S U(2)$ singlet. This fact is in contrast to the textbook SM, where the Higgs is part of a complex doublet and transforms alongside the GBs. Effective theories describing the Higgs as a part of a $S U(2)$ doublet $\Phi$ are termed Standard Model effective field theories (SMEFT).

The HEFT is fairly general and its form is largely independent of the details of the EWSBS because it is based only on the symmetry properties and the fact that only the light degrees of freedom are retained. The latter depends on the symmetry breaking pattern $G \rightarrow H$ and the way the electroweak gauge group $G_{\mathrm{EW}}$ is embedded in $G$. In this type of effective theories, the Higgs may or may not be a Goldstone boson. Having light states other than the Higgs (such as e.g., additional Goldstone bosons) could be worrisome from a phenomenological point of view because it would be very difficult or impossible to find mechanisms that would make them so massive to be able to escape detection. We should then exclude such a possibility from the effective theory.

When can a particular HEFT be written in the form of a SMEFT? Or in other words, when can a particular HEFT can be written in terms of the $S U(2)$ doublet $\Phi$ ? The answer is the following [46]; given some four-dimensional HEFT scalar manifold with metric $g_{\alpha \beta}(\omega)$ (with $h=\omega^{4}$ ), it is possible to find a field reparametrization so that the Lagrangian can be written in terms of the doublet $\Phi$ whenever there exists a $S U(2)_{L} \times S U(2)_{R}$ invariant point on the coset $G / H$. This is not always the case, but it happens in the SM.

The nonlinearity shows up as momentum-dependent vertices with an arbitrary high number of Goldstone boson insertions coming from the expansion of the matrix field

$$
U=\exp \left(\frac{i \omega^{a} \sigma^{a}}{v}\right) \approx 1+i \frac{\omega^{a} \sigma^{a}}{v}+O\left(\frac{\omega}{v}\right)^{2},
$$

where $\omega=\left\{\omega^{1}, \omega^{2}, \omega^{3}\right\}$ and $\sigma^{a}$ represents the $S U(2)$ Pauli matrices. The range of validity of the HEFT itself is given by the parameter controlling the expansion and sets a cutoff for the theory at $\Lambda=4 \pi v \approx 3 \mathrm{TeV}$. Given this, we would expect the resonances to emerge at a scale of a few $\mathrm{TeV}$; values in principle reachable at the LHC but their detection is difficult by several reasons. The main one is that they are produced only in vector boson fusion, a process that is subdominant at the LHC. The fact that the couplings of these putative resonances to the EWSBS are a priori unknown is also a serious handicap. In addition, from previous work we know that the dynamical resonances in question are generically narrow and not very visible, particularly if the anomalous couplings do not differ too much from their SM values [10]. However, their appearance is generic and by now their existence in extensions of the EWSBS seems well established by various unitarization methods $[17,20]$. Yet, detailed studies indicate that their confirmation may need the full $3000 \mathrm{fb}^{-1}$ statistics at the LHC [19] if only leptonic decays (4l) are considered in the final states and about one order of magnitude less if decays of the vector bosons in two jets are analyzed too; $(2 j+2 l)$.

The terms of the chiral Lagrangian are organized by the chiral dimension of its local operators. This counts the number of masses and derivatives (momenta) and a piece of the Lagrangian of chiral dimension $d, \mathcal{L}_{d}$, will contribute to the process at order $\mathcal{O}\left(p^{d}\right)$. For our analysis with next to leading order (NLO) precision, we restrict ourselves to operators up to $\mathcal{O}\left(p^{4}\right)$. The set of operators that participate in (on-shell) $2 \rightarrow 2$ scattering processes and are invariant under charge conjugation and parity transformations $(\mathrm{CP})$, Lorentz, Lorentz invariant, gauge, and custodial symmetries are gathered in the following Lagrangians

$$
\begin{aligned}
\mathcal{L}_{2}= & -\frac{1}{2 g^{2}} \operatorname{Tr}\left(\hat{W}_{\mu \nu} \hat{W}^{\mu \nu}\right)-\frac{1}{2 g^{\prime 2}} \operatorname{Tr}\left(\hat{B}_{\mu \nu} \hat{B}^{\mu \nu}\right) \\
& +\frac{v^{2}}{4} \mathcal{F}(h) \operatorname{Tr}\left(D^{\mu} U^{\dagger} D_{\mu} U\right)+\frac{1}{2} \partial_{\mu} h \partial^{\mu} h-V(h),
\end{aligned}
$$

with the usual definitions 


$$
\begin{aligned}
U & =\exp \left(\frac{i \omega^{a} \sigma^{a}}{v}\right) \in S U(2)_{V}, \quad V_{\mu}=D_{\mu} U^{\dagger} U, \quad \mathcal{F}(h)=1+2 a\left(\frac{h}{v}\right)+b\left(\frac{h}{v}\right)^{2}+\ldots, \\
D_{\mu} U & =\partial_{\mu} U+i \hat{W}_{\mu} U, \quad \hat{W}_{\mu}=g \frac{\vec{W}_{\mu} \cdot \vec{\sigma}}{2}, \quad \hat{W}_{\mu \nu}=\partial_{\mu} \hat{W}_{\nu}-\partial_{\nu} \hat{W}_{\mu}+i\left[\hat{W}_{\mu}, \hat{W}_{\nu}\right], \\
V(h) & =\frac{1}{2} M_{h}^{2} h^{2}+\lambda_{3} v h^{3}+\frac{\lambda_{4}}{4} h^{4}+\ldots, \quad \mathcal{G}(h)=1+b_{1}\left(\frac{h}{v}\right)+b_{2}\left(\frac{h}{v}\right)^{2}+\ldots
\end{aligned}
$$

From the last operator in (3), and taking into account the definitions above, we will just need for this study the first term in the expansion of $\partial^{\nu} \mathcal{G}(h)$, so we define the new coupling $\zeta \equiv b_{1} \chi$. In what concerns the Higgs potential $V(h)$, we will parametrize the departures from the SM trilinear and quartic self-couplings using the parameters $d_{3,4}$ such that $\lambda_{3,4}=d_{3,4} \lambda$, with $\lambda$ being the only SM Higgs self-interaction $\lambda=M_{h}^{2} /\left(2 v^{2}\right)$ coupling.

The relevant HEFT for our processes up to NLO is then the sum of $\mathcal{L}_{2}, \mathcal{L}_{4}$ and the gauge fixing and Faddeev-Popov terms

$$
\mathcal{L}=\mathcal{L}_{2}+\mathcal{L}_{4}+\mathcal{L}_{\mathrm{GF}}+\mathcal{L}_{\mathrm{FP}}
$$

In the custodial limit and using an arbitrary gauge, the last two pieces are built using the following functions

$$
\begin{gathered}
f_{i}=\partial_{\mu} W_{i}^{\mu}-\frac{g v \xi}{2} \omega_{i}+\ldots \quad i=1,2,3, \\
\mathcal{L}_{\mathrm{GF}}=-\frac{1}{2 \xi}\left(\sum_{i=1}^{3} f_{i}^{2}\right), \quad \mathcal{L}_{\mathrm{FP}}=\sum_{a, b=1}^{3} c_{a}^{\dagger} \frac{\delta f_{a}^{\prime}}{\delta \alpha_{b}} c_{b},
\end{gathered}
$$

where $f^{\prime}$ stands for the $S U(2)_{L} \times U(1)_{\mathcal{Y}}$ transformation of the function $f$ and $\alpha_{a}$ the gauge parameters.

We could enrich the HEFT with additional $S U(2)$ singlets $h^{1}(=h), h^{2}, h^{3}, \ldots$ and a term

$$
\frac{1}{2} g_{i j} \partial_{\mu} h^{i} \partial^{\mu} h^{j}-V\left(h^{1}, h^{2}, \ldots\right)
$$

This possibility will not be considered here. The interested reader can see [30] for more details.

TABLE I. Current experimental constraints on bosonic HEFT anomalous couplings at $95 \%$ C.L. See the text about the issue to extract the $a_{4}$ bound from the CMS analysis of [49].

\begin{tabular}{llc}
\hline \hline Couplings & Ref. & Experiments \\
\hline $0.89<a<1.13$ & {$[65]$} & LHC \\
$-0.76<b<2.56$ & {$[66]$} & ATLAS \\
$-3.3 \lambda<\lambda_{3}<8.5 \lambda$ & {$[54]$} & CMS \\
$\left|a_{1}\right|<0.004$ & {$[67]$} & LEP $(S$-parameter) \\
$-0.06<a_{2}-a_{3}<0.20$ & {$[68]$} & LEP and LHC \\
$-0.0061<a_{4}<0.0063$ & {$[48]$} & CMS (from $W Z \rightarrow 4 l)$ \\
$\left|a_{5}\right|<0.0008$ & {$[49]$} & CMS (from $W Z / W W \rightarrow 2 l 2 j$ ) \\
\hline \hline
\end{tabular}

The explicit gauge transformation is

$$
\hat{W}_{\mu}^{\prime}=g_{L} \hat{W}_{\mu} g_{L}^{\dagger}-\frac{1}{g} g_{L} \partial_{\mu} g_{L}^{\dagger}, \quad U^{\prime}=g_{L} U
$$

with $g_{L}=\exp (i \vec{\alpha}(x) \vec{\tau} / 2), S U(2)$ matrix. The custodial transformation is

$$
U^{\prime}=g U g^{\dagger},
$$

where $g$ is a constant $S U(2)$ matrix.

The notation we use roughly follows the conventions of [16]. In [4] the reader may find a complete list of operators in the HEFT up to chiral dimension four. ${ }^{1}$ Only a subset of those are relevant to us. Even taking this into consideration, the Lagrangians (2) and (3) contain a number of free parameters. Not including these already well established by experiments, we have $a$ and $b$ in the $O\left(p^{2}\right)$ Lagrangian, and $a_{3}, a_{4}, a_{5}$, $\gamma, \delta, \eta$, and $\zeta$ in the $O\left(p^{4}\right)$ Lagrangian. In the SM, $a=b=1$ and the rest are identically zero. In addition, and of particular interest to us, we have $\lambda_{3}$ and $\lambda_{4}$ in the Higgs potential. We expect departures from the SM values at most of order $10^{-3}$, possibly less, in all the 'anomalous' couplings.

The experimental situation concerning these couplings is as follows. The situation has been summarized e.g., in [14]. The experimental bounds for the chiral coupling $a$ have been measured by ATLAS and CMS in the subprocess $h \rightarrow W W$ at $95 \%$ C.L. to be $0.89<a<1.13$. Also, the first experimental bounds on the chiral parameter $b$ have been set by ATLAS with the subprocess $h h \rightarrow W W$. The result of this analysis, that assumes the absence of new physics resonances, is $-1.02<b<2.71$. As we see, there are still large experimental uncertainties regarding the Higgs couplings to vector bosons. These uncertainties affect operators of chiral dimension two and are accordingly expected to be the most relevant ones.

The chiral couplings $a_{4}$ and $a_{5}$ have received a lot of attention in the past because, to a large extent they control the appearance of resonances in the vector-isovector and scalar-isoscalar channels, at least in the approximation were the ET is assumed to hold in its most strict version and the propagation of transverse modes is neglected. ${ }^{2}$ Using

\footnotetext{
${ }^{1}$ Some operators are redundant [6] once the bosonic basis together with the fermionic one is considered, and equations of motion are used.

${ }^{2}$ Actually we will see below that the operators and couplings that survive in this extreme ET limit, i.e., $g=0$, are most relevant.
} 
only the $8 \mathrm{TeV}$ data, in 2017 ATLAS [47] set the bounds $-0.024<a_{4}<0.030$ and $-0.028<a_{5}<0.033$. More recently, CMS [48] using the $13 \mathrm{TeV}$ data and only $4 l$ decays from $W Z$ scattering was able to set the bounds $-0.0061<a_{4}<0.0063,-0.0094<a_{5}<0.0098$, about three times better. In [49], CMS studies $2 j+2 l$ decays from both $W W$ and $W Z$ scatterings to set the rather stringent bound ${ }^{3}\left|a_{5}\right|<0.0008$.

The smallness of these values in the case of $a_{4}$ and $a_{5}$ justifies, for the range of energies where they have been applied, the use of a simple approach, without unitarization. Yet, small as they are, there is still room for new physics resulting from unitarization. For instance, this range still allows for the appearance of vector resonances in the range $1.5 \mathrm{TeV}<M_{V}<2.5 \mathrm{TeV}$, both for $a=1$ and $a=0.9$ [19].

As for the coupling $a_{3}$, its range of uncertainty is quite large and its influence on the location and properties of resonances in BSM physics has not been assessed yet as this requires a full computation and subsequent unitarization studies including transverse modes of the vector bosons. This will be presented below.

Concerning the Higgs potential parameters, there are not relevant bounds on $\lambda_{4}$ (i.e., on possible departures from the SM relation $\lambda_{4}=\lambda=M_{h}^{2} / 2 v^{2}$ ). In what concerns $\lambda_{3}$, and recalling the parametrization $\lambda_{3}=d_{3} \lambda$, some recent bounds have been obtained by ATLAS [53], $-2.3<d_{3}<10.3$, combining double and single- Higgs analysis at $95 \%$ C.L., and by CMS [54], $-3.3<d_{3}<8.5$, from the subprocess $H H \rightarrow b \bar{b} \gamma \gamma$. To our knowledge, there are no experimental studies on the $\mathcal{O}\left(p^{4}\right)$ chiral parameter $\zeta$. However, as we stress in our work, this parameter plays a role in the $W W$-scattering at one loop.

\footnotetext{
${ }^{3} \mathrm{CMS}$ does not provide results for $a_{4}$ and $a_{5}$ directly as the analysis relies on the SMEFT, where the Higgs is treated as a doublet and the operators contributing to the scattering of four $W$ are of dimension eight (unlike in the HEFT where they are of dimension four). The basis adopted is the one introduced in [50], namely $f_{S, 0} / \Lambda^{4}$ and $f_{S, 1} / \Lambda^{4}$. However, as was later noted in [51,52], a third operator containing four derivatives of the Higgs doublet, with coefficient $f_{S, 2} / \Lambda^{4}$, exists in the SMEFT and cannot be in general missed. In order to get $f_{S, 0} / \Lambda^{4}, f_{S, 1} / \Lambda^{4}$, and $f_{S, 2} / \Lambda^{4}$ one needs to measure $W W$ and $W Z$ final states. This was done in the $4 l$ analysis of CMS [48]. However in [49] $W W$ and $W Z$ are combined together and it is not possible to extract $f_{S, 2}$ and $f_{S, 0}$ separately. Note that only xthe sum of the operators corresponding to $f_{S, 0}$ and $f_{S, 2}$ is custodially invariant, but neither of them is. The sum matches the chiral operator multiplying $a_{4}$ [52] in the HEFT. Therefore, a valid comparison requires assuming $f_{S, 0}=f_{S, 2}$ and only then
}

$$
a_{4}=\left.\frac{v^{4}}{8} \frac{f_{S, 0}}{\Lambda^{4}}\right|_{f_{S, 2}=f_{S, 0}}
$$

On the other hand, $f_{S, 1}$ is custodially invariant and

$$
a_{5}=\frac{v^{4}}{16} \frac{f_{S, 1}}{\Lambda^{4}} .
$$

\section{TREE LEVEL CALCULATION OF THE RELEVANT $2 \rightarrow 2$ PROCESSES}

As mentioned in the introduction in order to implement a fair comparison with experiment, we are interested in obtaining unitary amplitudes for the following $2 \rightarrow 2$ processes with one loop precision; $W_{L} W_{L} \rightarrow W_{L} W_{L}$, $W_{L} W_{L} \rightarrow h h$, and $h h \rightarrow h h$. In the first case, the $I=0$, $J=0$ (weak) isospin and angular momentum projection will be of most interest to us, but we will actually provide results that can be used for any $I, J$ projection thanks to the relations resulting from the exact isospin symmetry present for $g^{\prime}=0$. For instance, provided that custodial symmetry remains exact, from the $W^{+} W^{-} \rightarrow Z Z$ amplitude it is possible to obtain all the remaining $W_{L}^{a} W_{L}^{b} \rightarrow W_{L}^{c} W_{L}^{d}$ ones thanks to the isospin relations (see e.g., [10] for details). From Bose and crossing symmetries,

$$
\begin{aligned}
\mathcal{A}^{a b c d}= & \delta^{a b} \delta^{c d} \mathcal{A}\left(p^{a}, p^{b}, p^{c}, p^{d}\right) \\
& +\delta^{a c} \delta^{b d} \mathcal{A}\left(p^{a},-p^{c},-p^{b}, p^{d}\right) \\
& +\delta^{a d} \delta^{b c} \mathcal{A}\left(p^{a},-p^{d}, p^{c},-p^{b}\right)
\end{aligned}
$$

which allows us to write

$$
\begin{aligned}
\mathcal{A}^{+-00} & =\mathcal{A}\left(p^{a}, p^{b}, p^{c}, p^{d}\right), \\
\mathcal{A}^{+-+-} & =\mathcal{A}\left(p^{a}, p^{b}, p^{c}, p^{d}\right)+\mathcal{A}\left(p^{a},-p^{c},-p^{b}, p^{d}\right), \\
\mathcal{A}^{++++} & =\mathcal{A}\left(p^{a},-p^{c},-p^{b}, p^{d}\right)+\mathcal{A}\left(p^{a},-p^{d}, p^{c},-p^{b}\right) .
\end{aligned}
$$

This means that every amplitude with vector bosons as asymptotic states can be obtained by crossings from the fundamental amplitude $W^{+} W^{-} \rightarrow Z Z$, as mentioned before. Notice that crossing when longitudinally-polarized gauge bosons are involved has to be implemented via the momenta and not via Mandelstam variables because the polarization vectors do not transform covariantly (see e.g., the discussion in [10]).

The fixed isospin projections $T_{I}$ are given by

$$
\begin{aligned}
& T_{0}=3 \mathcal{A}^{+-00}+\mathcal{A}^{++++}, \\
& T_{1}=2 \mathcal{A}^{+-+-}-2 \mathcal{A}^{+-00}-\mathcal{A}^{++++}, \\
& T_{2}=\mathcal{A}^{++++} .
\end{aligned}
$$

Taking into account that in this framework the Higgs is a singlet, we can also write the projections for the crossed channels with an $I=0$ external state and the corresponding isospin amplitudes

$$
\begin{aligned}
\mathcal{A}\left(W_{L}^{a} W_{L}^{b} \rightarrow h h\right) & =\mathcal{A}^{a b}\left(p^{a}, p^{b}, p_{h, 1}, p_{h, 2}\right), \\
T_{W h, 0} & =\sqrt{3} \mathcal{A}^{+-},
\end{aligned}
$$




$$
\mathcal{A}(h h \rightarrow h h)=\mathcal{A}\left(p_{h, 1}, p_{h, 2}, p_{h, 3}, p_{h, 4}\right)=T_{h h, 0},
$$

where the last amplitude has obviously only an $I=0$ projection.

All the tree-level amplitudes gathered above have both leading order (LO) (computed using the Feynman rules from $\mathcal{L}_{2}$ ) and the NLO contributions (obtained using the rules of $\mathcal{L}_{4}$ ).

Below we present the tree-level amplitudes for the different $2 \rightarrow 2$ processes that are important for our study.
We use the following notation; a superindex indicates the different processes labeled as $W W$ for $W^{+} W^{-} \rightarrow Z Z, W h$ for $W^{+} W^{-} \rightarrow h h$, and $h h$ for $h h \rightarrow h h$. Also, each amplitude carries a subindex $x y$ that represents a process with a particle $y$ propagating in the $x$ channel. In the case with $x=c$ and no $y, \mathcal{A}_{c}$ represents the contact interaction of the four external particles. For instance, the amplitude $\mathcal{A}_{s h}^{W W}$ represents a Higgs exchanged in the $s$-channel of $W^{+} W^{-} \rightarrow Z Z$ scattering.

\section{A. $W^{+} W^{-} \rightarrow Z Z$}

The tree-level amplitude includes contribution from the $\mathcal{O}\left(p^{2}\right)$ and $\mathcal{O}\left(p^{4}\right)$ Lagrangian

$$
\begin{aligned}
\mathcal{A}_{c}^{W W}= & g^{2}\left(\left(\left(-2 a_{3}+a_{4}\right) g^{2}+1\right)\left(\left(\varepsilon_{1} \varepsilon_{4}\right)\left(\varepsilon_{2} \varepsilon_{3}\right)+\left(\varepsilon_{1} \varepsilon_{3}\right)\left(\varepsilon_{2} \varepsilon_{4}\right)\right)+2\left(\left(2 a_{3}+a_{5}\right) g^{2}-1\right)\left(\varepsilon_{1} \varepsilon_{2}\right)\left(\varepsilon_{3} \varepsilon_{4}\right)\right) \\
\mathcal{A}_{s h}^{W W}= & -\frac{a^{2} g^{2} M_{W}^{2}\left(\varepsilon_{1} \varepsilon_{2}\right)\left(\varepsilon_{3} \varepsilon_{4}\right)}{\left(p_{1}+p_{2}\right)^{2}-M_{H}^{2}}+\frac{a g^{4} \zeta}{4\left(\left(p_{1}+p_{2}\right)^{2}-M_{H}^{2}\right)}\left[2\left(\varepsilon_{3} \varepsilon_{4}\right)\left(\left(p_{1} \varepsilon_{2}\right)\left(p_{2} \varepsilon_{1}\right)-\left(\varepsilon_{1} \varepsilon_{2}\right)\left(p_{1}+p_{2}\right)^{2}\right)+2\left(\varepsilon_{1} \varepsilon_{2}\right)\left(p_{3} \varepsilon_{4}\right)\left(p_{4} \varepsilon_{3}\right)\right] \\
\mathcal{A}_{t W}^{W W}= & -\frac{\left(1-2 a_{3} g^{2}\right) g^{2}}{\left(p_{1}-p_{3}\right)^{2}-M_{W}^{2}}\left[-4\left(\left(\varepsilon_{1} \varepsilon_{2}\right)\left(p_{1} \varepsilon_{3}\right)\left(p_{2} \varepsilon_{4}\right)+\left(\varepsilon_{1} \varepsilon_{4}\right)\left(p_{1} \varepsilon_{3}\right)\left(p_{4} \varepsilon_{2}\right)+\left(\varepsilon_{2} \varepsilon_{3}\right)\left(p_{3} \varepsilon_{1}\right)\left(p_{2} \varepsilon_{4}\right)+\left(\varepsilon_{3} \varepsilon_{4}\right)\left(p_{3} \varepsilon_{1}\right)\left(p_{4} \varepsilon_{2}\right)\right)\right. \\
& +2\left(\left(\varepsilon_{2} \varepsilon_{4}\right)\left(\left(p_{1} \varepsilon_{3}\right)\left(p_{2}+p_{4}\right) \varepsilon_{1}+\left(p_{3} \varepsilon_{1}\right)\left(p_{2}+p_{4}\right) \varepsilon_{3}\right)+\left(\varepsilon_{1} \varepsilon_{3}\right)\left(\left(p_{2} \varepsilon_{4}\right)\left(p_{1}+p_{3}\right) \varepsilon_{2}+\left(p_{4} \varepsilon_{2}\right)\left(p_{1}+p_{3}\right) \varepsilon_{4}\right)\right) \\
& \left.-\left(\varepsilon_{1} \varepsilon_{3}\right)\left(\varepsilon_{2} \varepsilon_{4}\right)\left(\left(p_{1}+p_{3}\right) p_{2}+\left(p_{2}+p_{4}\right) p_{1}\right)\right] \\
\mathcal{A}_{u W}^{W W}= & \mathcal{A}_{t W}\left(p_{3} \leftrightarrow p_{4}, \varepsilon_{3} \leftrightarrow \varepsilon_{4}\right)
\end{aligned}
$$

where $\varepsilon_{i}$ is the abbreviation for $\varepsilon_{L}\left(p_{i}\right)$.

\section{B. $W_{L} W_{L} \rightarrow h h$}

$$
\begin{aligned}
\mathcal{A}_{c}^{W h}= & \frac{g^{2} b}{2}\left(\varepsilon_{1} \varepsilon_{2}\right)-\frac{g^{2} \eta}{v^{2}}\left(\left(\varepsilon_{1} p_{4}\right)\left(\varepsilon_{2} p_{3}\right)+\left(p_{3} \varepsilon_{1}\right)\left(\varepsilon_{2} p_{4}\right)\right)-\frac{2 g^{2} \delta}{v^{2}}\left(p_{3} p_{4}\right)\left(\varepsilon_{1} \varepsilon_{2}\right)+\frac{g^{2} \zeta}{v^{2}}\left(\left(\varepsilon_{1} \varepsilon_{2}\right)\left(p_{1}+p_{2}\right)^{2}-2\left(p_{1} \varepsilon_{2}\right)\left(p_{2} \varepsilon_{1}\right)\right), \\
\mathcal{A}_{s h}^{W h}= & \frac{3 g^{2} M_{h}^{2}}{2\left(\left(p_{1}+p_{2}\right)^{2}-M_{h}^{2}\right)}\left(a\left(\varepsilon_{1} \varepsilon_{2}\right)+\frac{\zeta}{v^{2}}\left(\left(\varepsilon_{1} \varepsilon_{2}\right)\left(p_{1}+p_{2}\right)^{2}-2\left(p_{1} \varepsilon_{2}\right)\left(p_{2} \varepsilon_{1}\right)\right)\right), \\
\mathcal{A}_{t \omega}^{W h}= & \frac{2 a^{2} g^{2}+a \zeta g^{4}}{2\left(p_{1}-p_{3}\right)^{2}}\left(\left(p_{3} \varepsilon_{1}\right)\left(p_{4} \varepsilon_{2}\right)\right), \\
\mathcal{A}_{t W}= & \frac{a^{2} g^{2} M_{W}^{2}}{\left(\left(p_{1}-p_{3}\right)^{2}-M_{W}^{2}\right)}\left(\varepsilon_{1} \varepsilon_{2}+\frac{\left(p_{4} \varepsilon_{2}\right)\left(\varepsilon_{1} p_{3}\right)}{\left(p_{1}-p_{3}\right)^{2}}\right)+\frac{a g^{4} \zeta}{2\left(\left(p_{1}-p_{3}\right)^{2}-M_{W}^{2}\right)}\left(2 M_{h}^{2}\left(\varepsilon_{1} \varepsilon_{2}\right)\right. \\
& \left.-\left(p_{4} \varepsilon_{2}\right)\left(p_{2} \varepsilon_{1}\right)-\left(\varepsilon_{1} p_{3}\right)\left(\varepsilon_{2} p_{3}\right)+M_{W}^{2} \frac{\left(p_{4} \varepsilon_{2}\right)\left(\varepsilon_{1} p_{3}\right)}{\left(p_{1}-p_{3}\right)^{2}}\right), \\
\mathcal{A}_{u \omega}^{W h}= & \mathcal{A}_{t \omega}^{W h}\left(p_{3} \leftrightarrow p_{4}\right), \\
\mathcal{A}_{u W}^{W h}= & \mathcal{A}_{t W}^{W h}\left(p_{3} \leftrightarrow p_{4}\right) .
\end{aligned}
$$

\section{C. $h h \rightarrow h h$}

$$
\begin{aligned}
& \mathcal{A}_{c}^{h h}=\frac{8 \gamma}{v^{4}}\left(\left(p_{1} p_{4}\right)\left(p_{2} p_{3}\right)+\left(p_{1} p_{3}\right)\left(p_{2} p_{4}\right)+\left(p_{1} p_{2}\right)\left(p_{3} p_{4}\right)\right)-6 \lambda_{4}, \\
& \mathcal{A}_{s h}^{h h}=-\frac{36 \lambda_{3}^{2} v^{2}}{\left(p_{1}+p_{2}\right)^{2}-M_{h}^{2}}, \\
& \mathcal{A}_{t h}^{h h}=\mathcal{A}_{s h}^{h h}\left(p_{2} \leftrightarrow-p_{3}\right), \\
& \mathcal{A}_{u h}^{h h}=\mathcal{A}_{s h}^{h h}\left(p_{2} \leftrightarrow-p_{4}\right) .
\end{aligned}
$$




\section{Counterterms}

The divergences eventually appearing in all these processes at the one-loop level have to be absorbed by redefining the parameters appearing at tree level. Namely,

$$
\begin{aligned}
v^{2} \rightarrow v^{2}+\delta v_{\mathrm{div}}^{2}+\delta \overline{v^{2}}, \quad\{h, \omega\} \rightarrow Z_{h, \omega}\{h, W, \omega\}, \quad M_{h, W}^{2} \rightarrow M_{h, W}^{2}+\delta M_{h, W}^{2}, \\
\lambda_{3,4} \rightarrow \lambda_{3,4}+\delta \lambda_{3,4}, \quad a \rightarrow a+\delta a, \quad b \rightarrow b+\delta b, \quad a_{i} \rightarrow a_{i}+\delta a_{i}, \\
\delta \rightarrow \delta+\delta \delta, \quad \eta \rightarrow \eta+\delta \eta, \quad \gamma \rightarrow \gamma+\delta \gamma, \quad \zeta \rightarrow \zeta+\delta \zeta,
\end{aligned}
$$

where we recall that $\zeta \equiv \chi b_{1}$.

Even though the gauge coupling $g$ appears in some of the previous formulas, the relation $M_{W}=g v / 2$ is assumed to all orders and the renormalization of $g$ is fixed by the ones of $v$ and $M_{W}$. On the contrary, we cannot assume the SM relation $M_{h}^{2}=2 v^{2} \lambda$ because this already assumes the persistence of the SM Higgs potential-something that we want to eventually test. It is for this reason that we keep separate notations $\lambda_{3}$ and $\lambda_{4}$ for the three- and four-point Higgs vertices.

In general all counterterms have both a divergent and a finite part, determined by the renormalization conditions. However, for reasons that will be clear later, we have split the counterterms for $v^{2}$ explicitly into divergent and finite pieces.

As we will see subsequently, we will determine all counterterms for processes involving only Goldstone bosons, whose calculation is substantially simpler than using vector bosons. This is enough to get all the necessary counterterms. The corresponding tree-level amplitudes for the Goldstones will be given in the next section.

\section{E. Auxiliary processes: $h \rightarrow \omega \omega, h \rightarrow h h$ and $h \rightarrow W \omega$}

In this subsection we collect a series of $1 \rightarrow 2$ tree-level processes that are useful to uniquely determine the counterterms. One of the processes $(h \rightarrow h h)$ cannot take place on shell, but it has to be rendered finite through the renormalization procedure. They are

(i) $h \rightarrow \omega \omega$ process. The tree-level amplitude of this decay up to NLO is (with $p_{h}$ the Higgs four-moment)

$$
\mathcal{A}_{\text {tree }}^{h \rightarrow \omega \omega}=-\frac{a p_{h}^{2}}{v},
$$

which leads to the on-shell renormalization condition

$$
\frac{M_{h}^{2}}{2 v^{3}}\left(a \delta v^{2}-2 v^{2} \delta a\right)+\operatorname{div}\left(\mathcal{A}_{1-\text { loop }}^{h \rightarrow \omega \omega}\right)=0 .
$$

From (19) and with the substitutions that will be specified later, we find the relation between $\delta a$ and $\delta v^{2}$, being the counterterms associated to the chiral parameter $a$ and to the vacuum expectation value (vev), respectively. Note that obviously $p_{h}$ does not get a counterterm even though on shell, $p_{h}^{2}=M_{h}^{2}$. (ii) $h \rightarrow h h$ process. At tree level, the corresponding amplitude reads

$$
\mathcal{A}_{\text {tree }}^{h \rightarrow h h}=-6 \lambda_{3} v .
$$

From the cancellation of the divergences of this process at one loop, we get a relation between $\delta \lambda_{3}$ and $\delta v^{2}$

$$
-\frac{3}{2 v^{3}}\left(d_{3} M_{h}^{2} \delta v^{2}+4 v^{4} \delta \lambda_{3}\right)+\operatorname{div}\left(\mathcal{A}_{1-\text { loop }}^{h \rightarrow h h}\right)=0 .
$$

Note that this (off-shell) process cannot be modified by using the equation of motion for $h$.

(iii) $h \rightarrow W \omega$ process

$$
\mathcal{A}_{\text {tree }}^{h \rightarrow W \omega}=i g\left(a+\frac{M_{W}^{2} \zeta}{v^{2}}\right) \varepsilon_{W} p_{h} .
$$

From the cancellation of the divergences of this process at one-loop level and with the assumption that the relation $M_{W}=\frac{1}{2} g v$ is satisfied at every order, we obtain a relation among $\delta v^{2}, \delta M_{W}^{2}, \delta a$, and $\delta \zeta$,

$$
\begin{aligned}
- & i\left(a M_{W}^{2} \delta v^{2}-2 M_{W}^{4} \delta \zeta-a v^{2} \delta M_{W}^{2}\right. \\
& \left.-2 M_{W}^{2} v^{2} \delta a\right) \frac{\varepsilon_{W} p_{h}}{M_{W} v^{3}}+\operatorname{div}\left(\mathcal{A}_{1-\text { loop }}^{h \rightarrow W \omega}\right)=0 .
\end{aligned}
$$

\section{ONE LOOP CALCULATION OF THE RELEVANT $2 \rightarrow 2$ PROCESSES AND COUNTERTERMS}

In this section we present the one loop calculation of the relevant amplitudes. The amplitudes cannot be expressed in terms of elementary functions as they are given by Passarino-Veltman integrals and they are quite cumbersome. For this reason we just show here the divergent parts (only present in the real part of the amplitude) and the explicit expression for the counterterms.

The calculation of quantum corrections for the processes requires gauge fixing and the inclusion of the FaddeevPopov ghosts. The results presented below will be given in the Landau gauge $\xi=0$. Obviously, physical amplitudes 
should be independent of the gauge choice, but some renormalization constants do depend on the gauge election. In the gauge-fixing processes several differences are present in the HEFT $[55,56]$ with respect to the textbook SM. On one hand, the Higgs is a singlet so it does not play any role in the symmetry and thus it is not present either in the gauge fixing piece, or in the Faddeev-Popov one, i.e., there are no Higgs-ghost interactions. On the other hand, the gauge condition in (6) translates into ghost-antighost pairs coupled to an arbitrary number of Goldstone insertions with a strength depending on the gauge parameter.

As mentioned before, the calculation of the $2 \rightarrow 2$ amplitudes we are interested in is relatively involved, even at the one-loop level. Recall that we will be interested both in the divergent part (to determine counterterms) but also in the much more involved finite part. This is particularly so because there are several free parameters that have to be considered when one moves away from the SM. For this reason it has become customary starting with the work of [10] to split the one-loop calculation into two parts. The imaginary part is computed exactly from the tree-level results described in Sec. III using the optical theorem, including only the $\mathcal{O}\left(p^{2}\right)$ pieces. The real part is computed making use of the equivalence theorem, replacing the longitudinal vector bosons in the external legs with the corresponding Goldstone bosons. However, the full set of polarizations (including of course transverse modes) will be kept internally inside the loops in the present study. We emphasize that this procedure is done only for efficiency reasons and there is no fundamental reason to do so.

Two reasons for concern might arise if this splitting between the real and imaginary parts is used. The first one is whether this actually preserves unitarity for unitarized amplitudes. A reassuring check will be presented in Sec. V, but the verification is actually guaranteed because the ET is working quite accurately provided that $s \gg M_{W}^{2}$, which is the regime we are actually interested in. In any case, unitarity should not be confused with the concept of 'perturbative unitarity' that relates the real and imaginary part up to a given order in perturbation theory and that only implies the consistency of the calculation in a field theory (even if this theory is nonunitary) and it is therefore automatic and of no interest to us; we have nevertheless verified that perturbative unitarity is well reproduced at the level of a few per cent as a check of the calculation by comparing the imaginary parts obtained in either way.

A second concern could be whether gauge invariance is preserved by doing this splitting. The answer is obviously in the affirmative in the following sense. The ET is derived from gauge invariance by requiring that in and out states fulfill the gauge condition (see e.g., [57]). A precise implementation of the ET tells us that corrections to the leading term (i.e., the one where the longitudinal gauge boson amplitude is approximated by the corresponding Goldstone boson scattering) are given by a succession of subleading contributions, each one lower with respect to the previous by a power of momenta. ${ }^{4}$ When translated into partial waves this implies that each of these successive corrections is suppressed by one more power of $s$. Taking into account that the one-loop amplitude is nominally of order $s^{2}$, corrections might change the $O(s)$ contribution only. However, the $O(s)$ contribution to the amplitude is computed exactly, without appealing to the ET. Therefore gauge invariance is guaranteed at the order we are computing. Having said that, it is safe to use the 't Hooft-Landau gauge which considerably simplifies the calculation. Where a comparison can be made, all counterterms agree with those computed in a general gauge as we will see below.

\section{A. Real part: The equivalence theorem}

The ET states that at high energies compared to the electroweak scale, the longitudinal projection of the vector boson can be substituted by the associated Goldstone boson allowing an error

$$
\varepsilon_{L}^{\mu}(k)=\frac{k^{\mu}}{M_{W}}+\mathcal{O}\left(\frac{M_{W}}{\sqrt{s}}\right) .
$$

This error assumed at the $\mathrm{TeV}$ scale, the cutoff of our theory, is then, nominally, lower than $10 \%$ but actually much lower because $M_{W}$ can appear only quadratically.

The calculation carried out in Ref. [15] just allowed the longitudinal part of the gauge bosons running inside the loops but for this study a full $\mathcal{O}(\mathrm{g})$ calculation is performed and the number of diagrams that needs to be taken into account scales to more that 1500 . This calculation has been done with the help of FeynArts [58], FeynCalc [59], and FeynHelpers [60] Mathematica packages. These routines are able to evaluate the one-loop integrals in the PassarinoVeltman notation [61] and extract just the divergent part of the diagrams when is required.

The expressions (10)-(13) are also valid within the equivalence theorem but now the symmetry will be manifest at the level of the Mandelstam variables themselves in the absence of polarization vectors that do not transform as four vectors under Lorentz transformations [16], which is a nice simplification.

\footnotetext{
${ }^{4}$ The ET relies on the splitting of the polarization vector $\epsilon_{L}^{\mu}=k^{\mu} / M_{W}+v^{\mu}$. Here $v^{\mu}$ is of order $M_{W} / E$. Substituting the splitting into the amplitude leads to corrections with higher and higher powers of $E$ in the denominator. When summed up they all reproduce the original $W_{L}$ amplitude. The reader can see [57] for details. We note that the ET is used here for the one-loop correction only, not for the tree -level contribution-different orders of $\hbar$. The one-loop correction to the partial wave is of $O\left(s^{2}\right)$ and the corrections implied by the ET might change the $O(s)$ contribution, but the latter-tree level-is calculated exactly without appealing to the ET. Therefore gauge invariance is respected even if the splitting is itself not gauge invariant.
} 
After use of the ET we have to consider the (real part of) the following processes.

$$
\text { 1. } \omega^{+} \omega^{-} \rightarrow z z
$$

From the isospin point of view, this is the fundamental amplitude for elastic $\omega \omega$ scattering. In this process 294 1PI diagrams participate at one-loop level. The divergences that appear need to be absorbed by redefinitions of coefficients of the tree-level amplitude up to NLO. When the $W_{L}$ are replaced by the $\omega$, following the equivalence theorem, the amplitude tree-level amplitude reads

$$
\begin{aligned}
\mathcal{A}_{\text {tree }}^{\omega \omega}= & -\frac{s\left(M_{h}^{2}-s\left(1-a^{2}\right)\right)}{\left(s-M_{h}^{2}\right) v^{2}}+\frac{4}{v^{4}}\left(a_{4}\left(t^{2}+u^{2}\right)+2 a_{5} s^{2}\right) \\
& +\left[\frac{g^{2}}{4} \frac{u-s}{t-M_{W}^{2}}\left(1+\frac{8 a_{3} t}{v^{2}}\right)+u \Leftrightarrow t\right]
\end{aligned}
$$

with the infinitesimal substitutions

$$
\begin{aligned}
M_{h}^{2} & \rightarrow M_{h}^{2}+\delta M_{h}^{2}, \quad M_{W}^{2} \rightarrow M_{W}^{2}+\delta M_{W}^{2}, \quad v^{2} \rightarrow v^{2}+\delta v^{2}, \\
a & \rightarrow a+\delta a, \quad a_{4} \rightarrow a_{4}+\delta a_{4}, \quad a_{5} \rightarrow a_{5}+\delta a_{5}, \quad a_{3} \rightarrow a_{3}+\delta a_{3} .
\end{aligned}
$$

Besides, a redefinition of the Goldstone fields in the Lagrangian needs to be used for the divergent corrections of the external legs

$$
\begin{gathered}
\left\{\omega^{ \pm}, z\right\} \rightarrow \sqrt{Z_{\omega^{ \pm}, z}}\left\{\omega^{ \pm}, z\right\} \approx\left(1+\frac{1}{2} \delta Z_{\omega^{ \pm}, z}\right)\left\{\omega^{ \pm}, z\right\} . \\
\text { 2. } \boldsymbol{\omega}^{+} \boldsymbol{\omega}^{-} \rightarrow \boldsymbol{h} \boldsymbol{h}
\end{gathered}
$$

This scattering requires computing 505 one-loop 1PI diagrams. The tree-level amplitude is

$$
\begin{aligned}
\mathcal{A}_{\text {tree }}^{\omega h}= & -b \frac{s}{v^{2}}-\frac{6 a \lambda_{3} s}{s-M_{h}^{2}}-\left[\frac{g^{2}}{4\left(t-M_{W}^{2}\right)}\left(2 a^{2} s+\frac{a^{2}}{t}\left(t-M_{h}^{2}\right)^{2}\right)+t \Leftrightarrow u\right] \\
& -\frac{1}{v^{2}}\left[\frac{\zeta a g^{2}}{2\left(t-M_{W}^{2}\right)}\left(t(s-u)+M_{h}^{4}\right)+t \Leftrightarrow u\right]-\frac{1}{v^{2}}\left[\frac{a^{2}}{t}\left(t-M_{h}^{2}\right)^{2}+t \Leftrightarrow u\right] \\
& +\frac{1}{v^{4}}\left(2 \delta s\left(s-M_{h}^{2}\right)+\eta\left(\left(t-M_{h}^{2}\right)^{2}+\left(u-M_{h}^{2}\right)\right)\right) .
\end{aligned}
$$

To get rid of the divergences of this process, the following substitutions for the couplings are needed

$$
\begin{gathered}
M_{h}^{2} \rightarrow M_{h}^{2}+\delta M_{h}^{2}, \quad M_{W}^{2} \rightarrow M_{W}^{2}+\delta M_{W}^{2}, \quad v^{2} \rightarrow v^{2}+\delta v^{2}, \quad a \rightarrow a+\delta a, \\
b \rightarrow b+\delta b, \quad \lambda_{3} \rightarrow \lambda_{3}+\delta \lambda_{3}, \quad \delta \rightarrow \delta+\delta \delta, \quad \eta \rightarrow \eta+\delta \eta, \quad \zeta \rightarrow \zeta+\delta \zeta .
\end{gathered}
$$

Now, apart from (28), we will also need the redefinition of the classical Higgs field

$$
h \rightarrow \sqrt{Z_{h}} h \approx\left(1+\frac{1}{2} \delta Z_{h}\right) h .
$$

\section{3. $h h \rightarrow h h$}

This process at the one-loop level contains 654 1PI diagrams and the divergences must be canceled from the parameters of the amplitude (17) once the usual Mandelstam definitions have been applied,

$$
\begin{aligned}
\mathcal{A}_{\text {tree }}^{\text {hh }}= & -6 \lambda_{4}-36 \lambda_{3}^{2} v^{2}\left(\frac{1}{s-M_{h}^{2}}+\frac{1}{t-M_{h}^{2}}+\frac{1}{u-M_{h}^{2}}\right) \\
& +\frac{8 \gamma}{v^{4}}\left(\left(\frac{s}{2}-M_{h}^{2}\right)^{2}\right. \\
& \left.+\left(\frac{t}{2}-M_{h}^{2}\right)^{2}+\left(\frac{u}{2}-M_{h}^{2}\right)^{2}\right) .
\end{aligned}
$$

The universal counterterms

$$
\begin{aligned}
M_{h}^{2} & \rightarrow M_{h}^{2}+\delta M_{h}^{2}, \quad v^{2} \rightarrow v^{2}+\delta v^{2}, \quad \lambda_{3} \rightarrow \lambda_{3}+\delta \lambda_{3}, \\
\lambda_{4} & \rightarrow \lambda_{4}+\delta \lambda_{4}, \quad \gamma \rightarrow \gamma+\delta \gamma,
\end{aligned}
$$


are required for absorbing the divergences, plus the Higgs redefinition (31).

\section{B. Determination of counterterms}

The real absorptive part has both finite and divergent parts. The divergences are reabsorbed in the amplitudes via new parameters from redefinitions of couplings and fields of the bare theory (5) given in the previous subsections.

The counterterms of our theory are not uniquely defined and depend on the choice of physical inputs to define the finite part of the amplitude. In this study the so-called onshell scheme (see e.g., [62]) has been used. It states that the physical mass is placed in the pole of the renormalized propagator with residue 1 . This means

$$
\begin{aligned}
\operatorname{Re}\left[\Pi_{h, W_{T}}\left(q^{2}=M_{h, W_{T}}^{2}\right)-\delta M_{h, W_{T}}^{2}\right] & =0, \\
\operatorname{Re}\left[\frac{d \Pi_{h, \omega, W}}{d q^{2}}\left(q^{2}=M_{h, W, \omega}^{2}\right)+\delta Z_{h, W, \omega}\right] & =0,
\end{aligned}
$$

where $\Pi\left(q^{2}\right)$ is the one-loop correction to the respective propagator. The on shell, first used in the context of LEP physics, has the advantage that many relevant radiative corrections involve only two-point functions. This is obvious for the masses and wave-function renormalization. After the splittings $\delta M_{h, W}^{2}=\delta \bar{M}_{h, W}^{2}+\delta M_{h, W \text {,div }}^{2}$, and $\delta Z_{h, \omega}=\delta \bar{Z}_{h, \omega}+\delta Z_{h, \omega, \text { div }}$ we obtain

$$
\begin{aligned}
\delta M_{h, \text { div }}^{2} & =\frac{\Delta}{32 \pi^{2} v^{2}}\left(3\left[6\left(2 a^{2}+b\right) M_{W}^{4}-6 a^{2} M_{W}^{2} M_{h}^{2}+\left(3 d_{3}^{2}+d_{4}+a^{2}\right) M_{h}^{4}\right]\right), \\
\delta M_{W, \text { div }}^{2} & =\frac{\Delta}{48 \pi^{2} v^{2}}\left(M_{W}^{2}\left[3\left(b-a^{2}\right) M_{h}^{2}+\left(-69+10 a^{2}\right) M_{W}^{2}\right]\right) \\
\delta Z_{h, \text { div }} & =\frac{\Delta}{16 \pi^{2} v^{2}}\left(3 a^{2}\left(3 M_{W}^{2}-M_{h}^{2}\right)\right) \\
\delta Z_{\omega, \text { div }} & =\frac{\Delta}{16 \pi^{2} v^{2}}\left(\left(b-a^{2}\right) M_{h}^{2}+3\left(a^{2}+2\right) M_{W}^{2}\right)
\end{aligned}
$$

where $\Delta \equiv \frac{1}{\epsilon}+\log (4 \pi)+\gamma_{E}$ and the dimensionality is set to $4+2 \epsilon$.

The one-loop level propagator mixing between the gauge boson and its associated Goldstone is protected by the gauge fixing condition in (6) and no extra counterterms will be needed for this. In the absence of electromagnetic interactions assuming an exact custodial symmetry, no $Z-\gamma$ mixing in the gauge propagator can occur either.

Besides, the condition of vanishing tadpole is assumed. There is an extra counterterm $\delta T$ that cancels the Higgs tadpole contribution at one loop satisfying the usual relation [15]

$$
\delta T=-v\left(\delta M_{h}^{2}-2 v^{2} \delta \lambda-2 \lambda \delta v^{2}\right)=-\mathcal{A}_{\mathrm{tad}}^{h} .
$$

With our parametrization for the Higgs potential, $\lambda$ does not appear in any of the processes but its counterterm can be determined using (36) once $\delta M_{h}^{2}$ and $\delta v^{2}$ are obtained.

The matrix field (1) containing the Goldstones in the HEFT should retain its unitarity and hence it cannot receive any multiplicative renormalization. Perturbatively, the redefinitions of the $n$-th term of the expansion of $U$ $\frac{1}{n !}\left(i \frac{\omega}{v}\right)^{n} \rightarrow \frac{1}{n !}\left(i \frac{\omega}{v}\right)^{n}+\frac{1}{2(n-1) !}\left(\delta Z_{\omega}-\frac{\delta v^{2}}{v^{2}}\right)\left(i \frac{\omega}{v}\right)^{n}$.

It turns out that to absorb the one-loop divergences, the counterterms for the Goldstone fields $\left(\sqrt{Z_{\omega}}\right)$ and the vev $\left(\sqrt{\delta v^{2}}\right)$ are equal so they cancel each other at every order in the expansion. The finite part of $\sqrt{\delta v^{2}}$ is fixed, at every order, by the condition

$$
\delta Z_{\omega}=\frac{\delta v^{2}}{v^{2}}
$$

The counterterms of the HEFT whose renormalization is not determined by the OS scheme conditions, are obtained in the $\overline{M S}$ scheme. Since this is a mass independent scheme, the counterterms corresponding to operators of dimension four (such as $a_{3}, a_{4}$, etc.) are independent of $M_{W}$.

The complete list of counterterms allowing us to get rid of the divergences of the three amplitudes in the previous subsections is 


$$
\begin{aligned}
\delta v_{\mathrm{div}}^{2}= & \frac{\Delta}{16 \pi^{2}}\left(\left(b-a^{2}\right) M_{h}^{2}+3\left(a^{2}+2\right) M_{W}^{2}\right), \quad \delta T_{\mathrm{div}}=-\frac{\Delta}{32 \pi^{2} v} 3\left(d_{3} M_{h}^{4}+6 a M_{W}^{4}\right), \\
\delta a= & \frac{\Delta}{32 \pi^{2} v^{2}}\left(6 a\left(-2 a^{2}+b+1\right) M_{W}^{2}+\left(5 a^{3}-a(2+3 b)-3 d_{3}\left(a^{2}-b\right)\right) M_{h}^{2}\right), \\
\delta b= & \frac{\Delta}{32 \pi^{2} v^{2}}\left(6\left(3 a^{4}-6 a^{2} b+b(b+2)\right) M_{W}^{2}-\left(21 a^{4}-a^{2}(8+19 b)+b(4+2 b)\right.\right. \\
& \left.\left.+6 a d_{3}\left(1+2 b-3 a^{2}\right)-3 d_{4}\left(b-a^{2}\right)\right) M_{h}^{2}\right), \\
\delta \lambda_{\mathrm{div}}= & \frac{\Delta}{64 \pi^{2} v^{4}}\left(\left(5 a^{2}-2 b+3\left(d_{3}\left(3 d_{3}-1\right)+d_{4}\right)\right) M_{h}^{4}-12\left(2 a^{2}+1\right) M_{W}^{2} M_{h}^{2}+18(a(2 a-1)+b) M_{W}^{4}\right), \\
\delta \lambda_{3}= & \frac{\Delta}{64 \pi^{2} v^{4}}\left(36 a b M_{W}^{4}+6\left(3 a^{3}-3 a b-d_{3}\left(5 a^{2}+1\right)\right) M_{W}^{2} M_{h}^{2}+\left(-9 a^{3}+3 a b+d_{3}\left(10 a^{2}-b\right)+9 d_{3} d_{4}\right) M_{h}^{4}\right), \\
\delta \lambda_{4}= & \frac{\Delta}{64 \pi^{2} v^{4}}\left(36 b^{2} M_{W}^{4}-12\left(a^{2}-b\right)\left(8 a^{2}-2 b-9 a d_{3}\right) M_{W}^{2} M_{h}^{2}\right. \\
& \left.+\left(96 a^{4}+4 b^{2}-d_{3}\left(114 a^{3}-42 a b\right)+9 d_{4}^{2}+a^{2}\left(-64 b+27 d_{3}^{2}+12 d_{4}\right)\right) M_{h}^{4}\right), \\
\delta a_{3}= & -\frac{\Delta}{384 \pi^{2}}\left(1-a^{2}\right), \quad \delta a_{4}=-\frac{\Delta}{192 \pi^{2}}\left(1-a^{2}\right)^{2}, \\
\delta a_{5}= & -\frac{\Delta}{768 \pi^{2}}\left(5 a^{4}-2 a^{2}(3 b+2)+3 b^{2}+2\right), \\
\delta \gamma= & -\frac{\Delta}{64 \pi^{2}} 3\left(b-a^{2}\right)^{2}, \quad \delta \delta=-\frac{\Delta}{192 \pi^{2}}\left(b-a^{2}\right)\left(7 a^{2}-b-6\right), \quad \delta \eta=-\frac{\Delta}{48 \pi^{2}}\left(b-a^{2}\right)^{2}, \\
\delta \zeta= & \frac{\Delta}{96 \pi^{2}} a\left(b-a^{2}\right) .
\end{aligned}
$$

For completeness we include the counterterm for $\delta g^{2}$ even though this is not an independent input of the theory anymore in the renormalization scheme used here,

$$
\delta g^{2}=g^{2}\left(\frac{\delta M_{W}^{2}}{M_{W}^{2}}+\frac{\delta v^{2}}{v^{2}}\right)=\frac{\Delta}{12 \pi^{2} v^{4}} M_{W}^{2}\left(\left(-51+19 a^{2}\right) M_{W}^{2}+6\left(b-a^{2}\right) M_{h}^{2}\right)
$$

Notice that our prescription is different from the usual one where one requires the renormalized $Z, \gamma$ two-point function to vanish at zero momentum. This condition cannot be implemented without electromagnetism, obviously. However, the different result for $\delta g$ is of no consequence in the on-shell scheme.

\section{Cross checks and comparison with previous results}

All these counterterms above have the correct SM limit. When $a=b=d_{3}=d_{4}=1$, all the parameters that are not present in the SM vanish, and we are left with $\delta v_{\text {div }}^{2}, \delta \lambda_{\text {div }}$, $\delta \lambda_{3}$, and $\delta \lambda_{4}$. In the SM limit, $\delta \lambda, \delta \lambda_{3}$, and $\delta \lambda_{4}$ have been checked to be exactly equal, as it should since they all derive from the unique SM Higgs potential coupling $\lambda$ present in the tadpole, triple, and quartic self-couplings. In particular

$$
\begin{aligned}
\delta \lambda_{\mathrm{div}, S M} & =\delta \lambda_{3, S M}=\delta \lambda_{4, S M} \\
& =\frac{\Delta}{16 \pi^{2} v^{4}} 3\left(3 M_{W}^{4}-3 M_{W}^{2} M_{h}^{2}+M_{h}^{4}\right) .
\end{aligned}
$$

As explained before, it can be seen just by direct comparison with (35), that the relation (38) is satisfied.

We can also compare our counterterms with the results previously reported in the literature. As mentioned before, the authors in Ref. [15] made a complete study of the elastic $\omega \omega$ scattering at one-loop level, allowing only longitudinal modes in the internal lines. That is, they set $g=0$ for the whole process and therefore they set the value $M_{W}=0$ for the vector boson mass. Our results (35) and (39) have been checked with those relevant for the process in [15] in the limit $M_{W}=0$.

A cruder approximation was taken in [63] where they studied all the processes including the $I=0$ final states but, besides setting $g=0$ and neglecting physical vector bosons in the loops, the authors took the limit $M_{h}=0$. In this limit where the self-interactions of the Higgs are absent, there is no need for redefinitions of $a, b$, and $v$ to absorb the oneloop divergences and we are left with $a_{4}, a_{5}, \gamma, \delta, \eta$. Our results agree with theirs in the limit $M_{W}=M_{h}=0$, so the inclusion of the transverse gauge modes does not modify these counterterms. 
We also find agreement with the results of [42], where the authors carried out the renormalization of the off-shell Green functions of the three processes studied in this work for the purely scalar sector of the custodial preserving HEFT with a light Higgs in the limit $g=0, g^{\prime}=0$ (i.e., $M_{W}=0$ ). For the comparison with our on-shell calculation, we have made use of the equations of motion for the Higgs and the Goldstone fields, omitting the leptonic contribution

$$
\begin{aligned}
& \square \omega=-\frac{2 a}{v} \partial_{\mu} \omega \partial^{\mu} h+\cdots, \\
& \square h=-V^{\prime}(h)+\left(\frac{a}{v}+\frac{b}{v^{2}} h\right) \partial_{\mu} \omega \partial^{\mu} \omega+\cdots,
\end{aligned}
$$

leading to the following redefinitions of the electroweak and chiral parameters.

$$
\begin{aligned}
M_{h}^{2} & =\tilde{M}_{h}^{2}-2 c_{\square H} \frac{\tilde{M}_{h}^{4}}{v^{2}}, \quad a=a_{C}+\frac{2 \tilde{M}_{h}^{2}}{v^{2}}\left(c_{7}-a_{C} c_{\square H}\right), \\
b & =b_{C}+2 \mu_{3}^{v}\left(c_{7}-a_{C} c_{\square H}\right)+\frac{\tilde{M}_{h}^{2}}{v^{2}}\left(8 a_{7}-8 a_{C} a_{\square H}-4 b_{C} c_{\square H}+a_{C} c_{\Delta H}\right), \\
\lambda_{3} & =\frac{\mu_{3}^{v}}{3 !}-\frac{\tilde{M}_{h}^{2}}{v^{2}} \mu_{3}^{v} c_{\square H}+\frac{\tilde{M}_{h}^{4}}{v^{4}}\left(\frac{1}{2} c_{\Delta H}-2 a_{\square H}\right), \\
\lambda_{4} & =\frac{\tilde{\lambda}}{3 !}-\left(\mu_{3}^{v}\right)^{2} c_{\square H}+\frac{\tilde{M}_{h}^{2}}{v^{2}}\left(\mu_{3}^{v}\left(\frac{5}{3} c_{\Delta H}-8 a_{\square H}\right)-\frac{4}{3} \tilde{\lambda}_{\square H}\right)+\frac{4 \tilde{M}_{h}^{4}}{v^{4}}\left(\frac{2}{3} a_{\Delta H}-b_{\square H}\right), \\
a_{4} & =c_{11}, \quad a_{5}=c_{6}-\frac{a_{C}}{2} c_{7}+\frac{a_{C}^{2}}{4} c_{\square H}, \quad \delta=-c_{20}+\frac{1}{2} a_{C} c_{\Delta H}, \\
\eta & =-c_{8}+2 a_{C} c_{10}-4 a_{C}^{2} c_{9}, \quad \gamma=c_{D H},
\end{aligned}
$$

where $\mu_{3}^{v} \equiv \frac{\mu_{3}}{v}$ and all the quantities of the form $\tilde{X}$ represent the parameters from their Lagrangian that have a direct counterpart in ours.

It is worth commenting on the relevance of off-shell calculations. First we see at once that the number of parameters simply explodes; trying to do phenomenology is in practice nearly impossible. Secondly, there is a large arbitrariness in using totally or partially the equations of motion so some degree of arbitrariness is unavoidable. Finally, we have to remember that off-shell couplings in an effective theory are devoid of any physical meaning. A glance at the lhs and the rhs of the previous equivalences should suffice to convince oneself that this is not the way to go.

We have also compared our results with the more recent study [41] where the authors performed a full (off-shell) renormalization of the one-loop Green functions involved in $W Z$ scattering (all polarizations considered) including custodially nonpreserving operators too. When we restrict our set of counterterms to those relevant for $W_{L} W_{L}$ scattering and take into account that custodially nonpreserving contributions are omitted, we find our results compatible with those of [41] with two differences originating from the inclusion by these authors of two $\mathcal{O}\left(p^{4}\right)$ operators,

$$
a_{\square \square} \frac{\square h \square h}{v^{2}}, \quad a_{\square V V} \frac{\square h}{v} \operatorname{Tr}\left(V_{\mu} V^{\mu}\right) .
$$

These operators can be reduced by using the equation of motion of the Higgs field at leading order in Eq. (42), and are for our purposes redundant. The first of these two operators enters directly in the renormalization of the propagator of the Higgs so, even with the same on-shell renormalization condition as ours, $\delta Z_{h}$ and $\delta M_{h}^{2}$ differ in a consistent way. After using the equations of motion, both operators are actually redundant and change the coefficients $M_{h}, a$, and $a_{5}$ following Eq. (43) with $a_{\square \square}=c_{\square H}$ and $a_{\square V V}=c_{7}$. As mentioned before, from this reference we are able to compare only those counterterms participating in our elastic $W_{L} W_{L}$ scattering within the custodial limit $\left(g^{\prime}=0, M_{Z}=M_{W}\right)$ and in the Landau gauge $(\xi=0)$ : $v^{2}, M_{h}^{2}, M_{W}^{2}, a, a_{3}, a_{4}, a_{5}$, and $\zeta$ (called $a_{d 2}$ in their notation).

$\delta T_{\text {div }}$ is also compatible up to a different sign in the renormalization condition for the Higgs tadpole. We do not find agreement, though, in the counterterm associated to the $S U(2)_{L}$ coupling $g$, coming from the fact that in our case it is a derived quantity as shown in (40).

One can check easily that all our additional or 'anomalous' counterterms do vanish in the SM limit, while this is not in general the case for the off-shell calculations in $[41,42]$.

The authors in [6] also obtained the divergences of the HEFT local operators with the heat kernel formalism for the path integral. To this purpose, they needed to make redefinitions of the quantum fields, in particular the 
Higgs field, so they were present in the canonical normalization. These redefinitions alter the UV divergences of some operators with respect to those in our Lagrangian. All the $\mathcal{O}\left(p^{4}\right)$ divergences, not affected by field renormalizations, have been checked to coincide after some reparametrization of the chiral couplings.

\section{Imaginary part: The optical theorem}

The imaginary part of the NLO amplitude is obtained exactly using the optical theorem. The fact that some states can go on shell in the process forces the presence of a physical cut in the analytic structure of an amplitude that depends on the variable $s$ promoted to a complex quantity. This amplitude is obtained after the analytical continuation to the whole complex plane of the Feynman amplitude depending on the center of mass energy, a real variable.

Given a physical amplitude $\mathcal{A}(s)$, once we know the discontinuity of the complex amplitude across the physical cut with the usual Cutkosky rules, we find

$$
\operatorname{Im} \mathcal{A}(s)=\sigma(s)|\mathcal{A}(s)|^{2},
$$

where $\sigma(s)=\sqrt{1-\frac{\left(M_{1}+M_{2}\right)^{2}}{s}}$ is the two-body phase space. This allows us to compute the imaginary part of any amplitude at the one-loop level from the tree-level result.

As an example, if we are interested in computing the full $I=1$ isospin amplitude in the process $W_{L}^{+} W_{L}^{-} \rightarrow Z_{L} Z_{L}$

$$
\mathcal{A}\left(W_{L}^{+} W_{L}^{-} \rightarrow Z_{L} Z_{L}\right)=\mathcal{A}_{\text {tree }}^{(2)}+\mathcal{A}_{\text {tree }}^{(4)}+\mathcal{A}_{\text {loop }}^{(4)},
$$

where $\mathcal{A}_{\text {tree }}^{(2)}+\mathcal{A}_{\text {tree }}^{(4)}$ is the amplitude (15) and $\mathcal{A}_{\text {loop }}^{(4)}$ is the full one-loop amplitude

$$
\mathcal{A}_{\text {loop }}^{(4)}=\operatorname{Re}\left[\mathcal{A}_{\text {loop }}^{(4)}\left(\omega^{+} \omega^{-} \rightarrow z z\right)\right]+i \sigma(s)\left|\mathcal{A}_{\text {tree }}^{(2)}\right|^{2} .
$$

This procedure is not necessary but speeds up the calculation.

\section{UNITARIZATION}

Departures from the SM such as those described by the HEFT unavoidably result in a loss of unitarity. Amplitudes typically exhibit a bad ultraviolet behavior leading to cross sections that grow too fast with the energy $\sqrt{s}$ and quickly violate the unitarity bounds. While this is well known, it is sometimes forgotten that this fast growth of the amplitudes results in a hypersensitivity to deviations of the coefficients of the HEFT with respect to their SM values. We analyze this in some more detail in the next subsections.

Once we have built the fixed isospin amplitudes using Eq. (12), we can obtain the amplitude with $J$ total angular momentum with the corresponding partial wave
$t_{I J}(s)=\frac{1}{32 K \pi} \int_{-1}^{1} d(\cos \theta) P_{J}(\cos \theta) T_{I}(s, \cos \theta)$,

using the center of mass relations $t=-\left(s-4 M_{W}^{2}\right)(1-$ $\cos \theta) / 2$ and $u=-\left(s-4 M_{W}^{2}\right)(1+\cos \theta) / 2 . K$ is a constant whose value is $K=2$ or 1 depending on whether the particles participating in the process are identical or not.

The way we compute the fixed isospin amplitudes using Feynman diagrams from the Lagrangian (5), leads to a perturbative expansion of the form

$$
t_{I J}(s) \approx t_{I J}^{(2)}+t_{I J}^{(4)}+\ldots,
$$

which for the $I, J=1,1$ case satisfies perturbatively the optical theorem (45)

$$
\begin{aligned}
& \operatorname{Im}\left(t_{11}^{(2)}\right)=0 \\
& \operatorname{Im}\left(t_{11}^{(4)}\right)=\sqrt{1-\frac{4 M_{W}^{2}}{s}\left|t_{11}^{(2)}\right|^{2}}
\end{aligned}
$$

\section{A. Amplitudes at high energies}

In Fig. 1 we plot the modulus computed at the tree plus one-loop level for the process $W_{L} W_{L} \rightarrow Z_{L} Z_{L}$ for various values of the parameter $a$ at a fixed scattering angle $\cos \theta=0.3$. Departures from the SM value $a=1$ result in a clear bad high-energy behavior. This same behavior is seen in the remaining $2 \rightarrow 2$ processes. For instance the modulus of the amplitude for the process $W_{L} W_{L} \rightarrow h h$ is depicted in the left panel of Fig. 2 for the same values of the

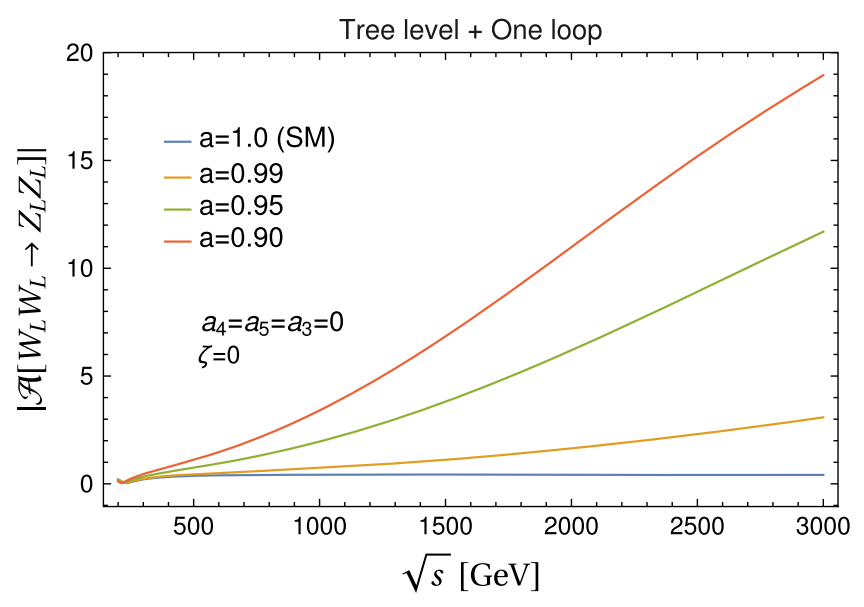

FIG. 1. Plot of the modulus of the elastic vector boson scattering amplitude in longitudinal polarization $\left(W_{L} W_{L} \rightarrow\right.$ $Z_{L} Z_{L}$ ) versus the center of mass energy $\sqrt{s}$ for some values of the chiral parameter $a$ at a fixed scattering angle $\cos \theta=0.3$. It can be seen how small departures for the SM value $(a=1)$ leads to a quick violation of unitarity within the HEFT regime of validity. All the $\mathcal{O}\left(p^{4}\right)$ couplings contributing to the process $\left(a_{3}, a_{4}, a_{5}, \zeta\right)$ are set to zero. 

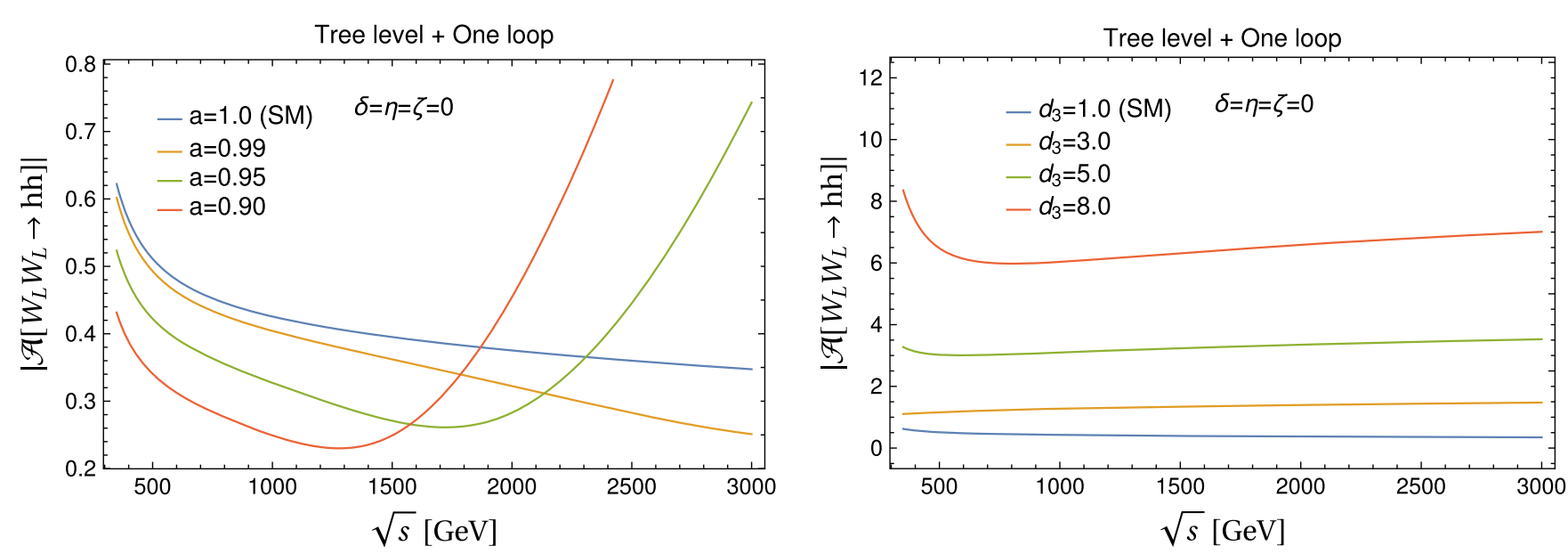

FIG. 2. Plot of the modulus of the $W_{L} W_{L} \rightarrow h h$ amplitude versus the center of mass energy $\sqrt{s}$ for some values of the chiral parameter $a$ (left) and the trilinear Higgs coupling $\lambda_{3}=d_{3} \lambda$ (right) at a fixed scattering angle $\cos \theta=0.3$. It can be seen how departures from the SM limit do not lead to an obvious bad UV behavior of the amplitude in the second case. Although it could seem that in the left panel there is no bad high-energy limit for the case $a=0.99$, the reality is that the amplitude acquires an unphysical behavior for a scale just above the cutoff of the theory (around $4 \mathrm{TeV}$ ), a region not shown in the plot. In each figure, the remaining parameters do not vary and are set to the corresponding SM values.

parameter $a$ parameterizing the Higgs-vector boson coupling in the HEFT.

On the contrary, this same modulus of the $W_{L} W_{L} \rightarrow h h$ amplitude (Fig. 2) shows a milder dependence on the parameter $\lambda_{3}$ of the Higgs potential. For the SM value $a=1$, modifying $\lambda_{3}$ does not show obvious signs of bad high-energy behavior. At this level, this derives from the fact that this coupling is momentum independent. Note that this coupling is very poorly constrained so the overall uncertainty of the amplitude is accordingly large.

Higher-loop calculations will only worsen the highenergy behavior. It is thus clear that, except for tiny deviations from the SM, as soon as one enters the multi $\mathrm{TeV}$ region, the perturbative treatment is unreliable. Therefore, checking for constraints on the anomalous couplings present in the HEFT by just looking at growing cross sections is risky but may be justified (if the deviations are small) or plain wrong (if the anomalous coupling constants deviate significantly from their SM values). It is clear that physical amplitudes - even beyond the SM-are necessarily unitary, meaning that in the HEFT higher-loop contributions have to be somehow summed up to render a reasonable high-energy behavior, which of course will be different from the SM one, but still in accordance with the general principles of field theory. We conclude that unitarization is necessary to compare the predictions of the HEFT with those of the SM vis-à-vis the experiments at very high energies, particularly when we are close to the HEFT UV cutoff.

The bad-energy behavior can also be seen at the partial wave level before unitarization. The modulus of the vectorisovector contribution up to the NLO in the expansion (49) is shown in Fig. 3 for a small departure from the SM values via the parameter $a_{4}=10^{-4}$ (the rest of the parameters are set to their corresponding SM values). In that same figure (in the right axis), the contribution in percentage (in absolute value) to the one-loop partial wave is shown with respect to the full tree level $\left[\mathcal{O}\left(p^{2}\right)+\mathcal{O}\left(p^{4}\right)\right]$ with the following definition

$$
\Delta_{1-\text { loop }}=100 \cdot\left|\frac{\left|t_{11}^{\text {tree }+ \text { loop }}\right|-\left|t_{11}^{\text {tree }}\right|}{\left|t_{11}^{\text {tree }}\right|}\right| .
$$

The contribution of the one-loop level to the full partial wave turns out to be negative as it can be seen in the figure (the green dot-dashed line representing the full amplitude is

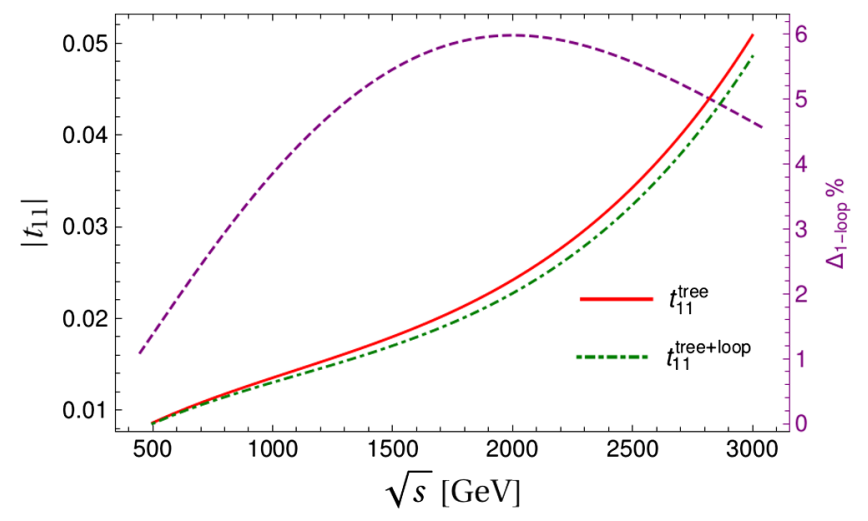

FIG. 3. (Left axis) Plot of the modulus of the vector-isovector partial wave at tree level (solid red line) and tree + one-loop level (dot-dashed green line) versus the center of mass energy $\sqrt{s}$. (Right axis) Plot of the percentage represented by the 1-loop contribution (purple dashed line), $\Delta_{1-\text { loop }}$ in Eq. (51) versus the center of mass energy $\sqrt{s}$ in absolute value. The curves are depicted for $a_{4}=10^{-4}$ and the rest of the parameters are set to their SM values. 
always below the tree level contribution in solid red), reaching a maximum value $\sim 6 \%$ around the $2 \mathrm{TeV}$ region. Here the tree level contribution includes, as mentioned, both the $O\left(p^{2}\right)$ and the $O\left(p^{4}\right)$ pieces. As was already notice in previous works [15], as soon as one departs from the SM the later quickly dominate the real part of the $O\left(p^{4}\right)$ contribution (yet another reason why using the ET is justified).

\section{B. The inverse amplitude method}

The expansion in terms of the external momentum typically leads very quickly to a violation of unitarity and, in order to make realistic predictions, unitarization techniques need to be used. In our case we choose to make use of the IAM [18,20,22-28] in order to unitarize the partial waves (and, eventually, the amplitudes). The IAM is really successful in predicting the features of the rho meson resonance studying low-energy QCD with pion-pion scattering and it has also been extensively used in HEFT analysis.

The method consists in building the following IAM amplitude up to NLO

$$
t_{I J}^{\mathrm{IAM}} \simeq \frac{\left(t_{I J}^{(2)}\right)^{2}}{t_{I J}^{(2)}-t_{I J}^{(4)}},
$$

which perturbatively satisfies (49) and the desired unitarity condition $t_{I J}(s)=\frac{i}{2}\left(1-\eta(s) e^{2 i \delta(s)}\right)$, where $0<\eta<1$ is the ineslasticity.

A pole in the unitary amplitude (52) appears when, for some complex value of $s_{R}$ (53),

$$
t_{I J}^{(2)}\left(s_{R}\right)-t_{I J}^{(4)}\left(s_{R}\right)=0 .
$$

This pole, if present, is interpreted as a resonance with quantum numbers $I, J$ and features $M_{R}$ and $\Gamma_{R}$, these lasts given a la Wigner by the position of the pole in the complex plane $s_{R}=\left(M_{R}-\frac{i}{2} \Gamma_{R}\right)^{2}$. We will consider for this study and future ones only the lowest partial wave in $I=0,1$ channels and refer to these resonances as scalar-isoscalar for the poles in $t_{00}^{\mathrm{IAM}}$ and vector-isovector for $t_{11}^{\mathrm{IAM}}$.

One nicety of the IAM, besides assuring unitarity, is that the poles can be interpreted as dynamically generated resonances appearing after the resummation of infinite bubbles chain $W W \rightarrow Z Z \rightarrow W W \rightarrow \ldots \rightarrow Z Z$ (in the $I=1$ channel) as it can be understood diagrammatically from the perturbative expansion of (52).

An example of the recovery of unitarized amplitudes by the IAM is depicted in Fig. 4. In that illustration we show both IAM and partial wave amplitudes for the two benchmark points defined in the next section; Table II, $B P 1^{\prime}$ and $B P 2^{\prime}$. It can be seen how the inclusion of the NLO contribution leads to an even quicker violation of the unitarity of the partial wave in the UV regime of the

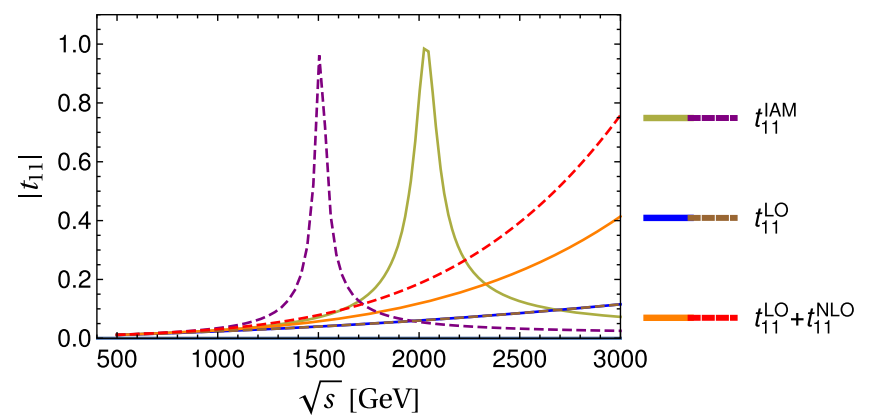

FIG. 4. Plot of the inverse amplitude method and partial wave amplitudes of the vector-isovector channel $I, J=1,1$ for the two benchmark points $\mathrm{BP}^{\prime}$ (dashed) and $\mathrm{BP} 2^{\prime}$ (solid) defined in Table II as a function of the center of mass energy $\sqrt{s}$. The notation of LO corresponds to the chiral order two $\left(t_{11}^{(2)}\right)$ and the NLO to the chiral order four $\left(t_{11}^{(4)}\right)$ partial waves in Eq. (49). Since the two benchmark points represented here share the value of $a$, the two LO lines, independent of the $\mathcal{O}\left(p^{4}\right)$ parameters, coincide.

theory. This unphysical high-energy behavior is tamed by means of the IAM amplitude defined in Eq. (52), exhibiting resonances for a BSM model. Figure 4 also shows the importance of the next to leading order versus the leading order contribution, reaching a 40\%-60\% relative size difference near the cutoff of the theory.

In the $I=0$ channel, two Higgs intermediate states are possible and for that one needs the machinery of coupled channels.

The IAM method can be extended to the coupled channel case too (see [25,64]), particularly if all the different channels have the same thresholds. From the perturbative expansion

$$
T_{I J}=T_{I J}^{(2)}+T_{I J}^{(4)}+\ldots
$$

a natural generalization of the IAM gives

$$
T_{I J}^{\mathrm{IAM}}=T_{I J}^{(2)}\left(T_{I J}^{(2)}-T_{I J}^{(4)}\right)^{-1} T_{I J}^{(2)},
$$

which satisfies exact multichannel elastic unitarity on the right cut

$$
\operatorname{Im} T_{I J}^{\mathrm{IAM}}=T_{I J}^{\mathrm{IAM}}\left(T_{I J}^{\mathrm{IAM}}\right)^{\dagger} .
$$

TABLE II. Values for the location of the vector poles $\sqrt{s_{V}}=$ $M_{V}-\frac{i}{2} \Gamma_{V}$ found in all the benchmark points of reference [19] once the transverse modes are included $(g \neq 0)$.

\begin{tabular}{lccccc}
\hline \hline$\sqrt{s_{V}}(\mathrm{GeV})$ & $g=0$ & $g \neq 0$ & $a$ & $a_{4} \cdot 10^{4}$ & $a_{5} \cdot 10^{4}$ \\
\hline BP1 & $1476-\frac{i}{2} 14$ & $1503-\frac{i}{2} 13$ & 1 & 3.5 & -3 \\
BP2 & $2039-\frac{i}{2} 21$ & $2087-\frac{i}{2} 20$ & 1 & 1 & -1 \\
BP3 & $2473-\frac{i}{2} 27$ & $2540-\frac{i}{2} 27$ & 1 & 0.5 & -0.5 \\
BP1 $^{\prime}$ & $1479-\frac{i}{2} 42$ & $1505-\frac{i}{2} 44$ & 0.9 & 9.5 & -6.5 \\
BP2' $^{\prime}$ & $1981-\frac{i}{2} 97$ & $2025-\frac{i}{2} 98$ & 0.9 & 5.5 & -2.5 \\
BP3' $^{\prime}$ & $2481-\frac{i}{2} 183$ & $2547-\frac{i}{2} 183$ & 0.9 & 4 & -1 \\
\hline \hline
\end{tabular}


The IAM has been extensively used to describe low-energy meson-meson scattering where it has proven to be extremely successful. With a very small set of parameters, it is able to describe many different channels including their first resonances $[17,20,25,64]$. In the case of coupled channels, the different amplitude matrix elements (partial waves) $\left(T_{I J}\right)_{i j}(s)$ correspond to different reactions having the same quantum numbers $I J$. Clearly, if there is a resonance in one of the channels it should appear also in all the others since physically these resonances can be produced in any of the reactions.

While for single-channel unitarization the IAM is well grounded and relies on a minimal set of assumptions (see e.g., $[17,21,25,64])$, there is no really unambiguous way of applying the IAM to the case where there are coupled channels with different thresholds. We shall adhere to the simplest choice that consists in assuming the previous expressions to remain valid also in the present analysis. This can be justified heuristically on the grounds that $M_{W}$ is not too different from $M_{h}$. This is again a good justification of the need to include all polarizations of the vector boson with a mass $M_{W}$ in the calculation.

In addition, it should be stated that the decoupling of the two $I=0$ channels in the case $a^{2}=b$ taking place when the equivalence theorem is used and physical $W_{L}$ are replaced by the corresponding Goldstone bosons does not hold in the exact calculation.

The results for the $I J=00$ channel will be reported in a separate publication. Here we will concentrate in the modifications that the inclusion of the transverse mode propagation of the vector bosons with a mass $M_{W}$ and the appearance of new effective couplings in the HEFT induce in the $I J=11$ channel.

\section{Vector resonances}

In order to see the relevance of including the propagation of transverse modes, we focus on vector resonances with quantum numbers $I, J=1,1$ in vector boson scattering (VBS). We shall compare the new results with those obtained previously.

From Eqs. (11) and (12), the fixed isospin amplitudes in the chiral expansion, $T_{1}^{(2)}$ and $T_{1}^{(4)}$ are obtained. $T_{1}^{(2)}$ using $\mathcal{A}_{\text {tree }}^{(2)}\left(p_{1}, p_{2}, p_{3}, p_{4}\right)$ and $T_{1}^{(4)}$ with $\mathcal{A}_{\text {tree }}^{(4)}\left(p_{1}, p_{2}, p_{3}, p_{4}\right)+$ $\operatorname{Re}\left[\mathcal{A}_{\text {loop }}\left(\omega^{+} \omega^{-} \rightarrow z z\right)\right]\left(p_{1}, p_{2}, p_{3}, p_{4}\right)$. Using Eqs. (48) and (50) perturbatively, we find the partial wave for $I, J=1,1$

$$
\begin{aligned}
t_{11}^{(2)} & =\frac{1}{64 \pi} \int_{-1}^{1} d(\cos \theta) \cos \theta T_{1}^{(2)}(s, \cos \theta), \\
\operatorname{Re}\left[t_{11}^{(4)}\right] & =\frac{1}{64 \pi} \int_{-1}^{1} d(\cos \theta) \cos \theta T_{1}^{(4)}(s, \cos \theta), \\
\operatorname{Im}\left[t_{11}^{(4)}\right] & =\sqrt{1-\frac{4 M_{W}^{2}}{s}}\left|t_{11}^{(2)}\right|^{2},
\end{aligned}
$$

where the Legendre polynomial $P_{1}(\cos \theta)=\cos \theta$ has been used.

The vector-isovector resonances, if present, are located by searching for poles of the unitary IAM amplitude (52) i.e., looking for solutions of Eq. (53).

Let us first of all investigate how the proper inclusion of the transverse modes (i.e., $g \neq 0$ ) influence the results obtained in the extreme ET limit. Below we provide results for $g=0$ and $g=2 M_{W} / v$. The benchmark points correspond to those used in [19]. As it can be seen ceteris paribus the inclusion of the gauge boson masses systematically increases the masses of the resonances by a few per cent. The modifications in the widths are not significant. In these calculations $b=a^{2}$, and both $a_{3}$ and $\zeta$ have been set to zero.

\section{Checking unitarity}

As a check of the good unitarity behavior of the amplitudes obtained in the IAM and the validity of the approximations made we plot the partial wave for complex values of the kinematical variable $s$ in the $I J=11$ channel. There are no threshold in this channel beyond the elastic channel and the results must lie accordingly in a circumference of radius $1 / 2$ centered at $s=i / 2$. This is shown in Fig. 5. We also plot the results obtained for the same $I J=11$ channel in perturbation theory-without resummation. They obviously violate the unitarity bound. The plot correspond to the values of the benchmark point $\mathrm{BP} 2^{\prime}$ from Table II, corresponding to $a=0.9, a_{4}=5.5 \times 10^{-4}$, and $a_{5}=-2.5 \times 10^{-4}$, within the recent CMS experimental bounds of Table I.

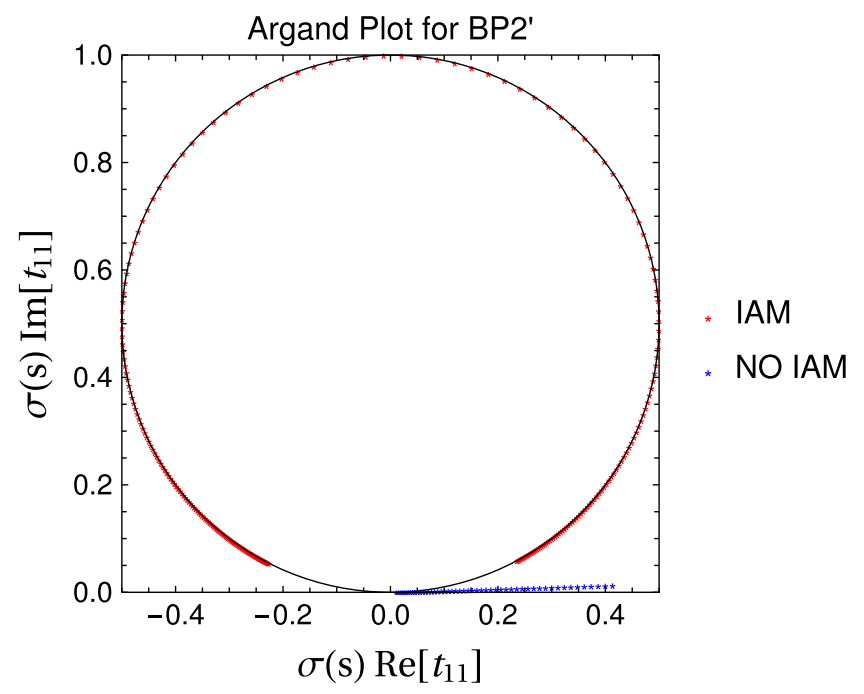

FIG. 5. Argand plot showing the unitary VBS amplitude (red points) for the values of BP2' from Table II. Due to the elasticity of the process, the IAM amplitude lies exactly on the unitarity limit, i.e., the circumference of radius $1 / 2$ centered at $(0,1 / 2)$. The amplitude before applying the IAM is also present (blue points) and obviously lies entirely outside the unitarity condition. 
TABLE III. Values for the location of the vector poles $\sqrt{s_{V}}=M_{V}-\frac{i}{2} \Gamma_{V}$ found in all the benchmark points of reference [19] for different values of $a_{3}$ and $g \neq 0$. The chiral parameter $\zeta$ is set to zero.

\begin{tabular}{lccccc}
\hline \hline$\sqrt{s_{V}}(\mathrm{GeV})$ & $a_{3}=0$ & $a_{3}=0.1$ & $a_{3}=-0.1$ & $a_{3}=0.01$ & $a_{3}=-0.01$ \\
\hline BP1 & $1503-\frac{i}{2} 13$ & $1795-\frac{i}{2} 11$ & $1215-\frac{i}{2} 15$ & $1532-\frac{i}{2} 13$ & $1474-\frac{i}{2} 13$ \\
BP2 & $2087-\frac{i}{2} 20$ & $2721-\frac{i}{2} 15$ & $1505-\frac{i}{2} 23$ & $2150-\frac{i}{2} 19$ & $2025-\frac{i}{2} 21$ \\
BP1 $^{\prime}$ & $1505-\frac{i}{2} 44$ & $1663-\frac{i}{2} 46$ & $1335-\frac{i}{2} 43$ & $1520-\frac{i}{2} 44$ & $1488-\frac{i}{2} 44$ \\
BP2 $^{\prime}$ & $2025-\frac{i}{2} 98$ & $2278-\frac{i}{2} 104$ & $1752-\frac{i}{2} 89$ & $2052-\frac{i}{2} 98$ & $1999-\frac{i}{2} 97$ \\
\hline \hline
\end{tabular}

TABLE IV. Values for location of the vector poles $\sqrt{s_{V}}=M_{V}-\frac{i}{2} \Gamma_{V}$ found in all the benchmark points of reference [19] for different values of $\zeta$ and $g \neq 0$. The chiral parameter $a_{3}$ is set to zero.

\begin{tabular}{lccccc}
\hline \hline$\sqrt{s_{V}}(\mathrm{GeV})$ & $\zeta=0$ & $\zeta=0.1$ & $\zeta=-0.1$ & $\zeta=0.01$ & $\zeta=-0.01$ \\
\hline BP1 & $1503-\frac{i}{2} 13$ & $1637-\frac{i}{2} 13$ & $1377-\frac{i}{2} 14$ & $1516-\frac{i}{2} 13$ & $1489-\frac{i}{2} 13$ \\
BP2 & $2087-\frac{i}{2} 20$ & $2393-\frac{i}{2} 18$ & $1809-\frac{i}{2} 22$ & $2117-\frac{i}{2} 20$ & $2058-\frac{i}{2} 21$ \\
BP1 $^{\prime}$ & $1505-\frac{i}{2} 44$ & $1570-\frac{i}{2} 46$ & $1439-\frac{i}{2} 43$ & $1510-\frac{i}{2} 45$ & $1497-\frac{i}{2} 45$ \\
BP2 $^{\prime}$ & $2025-\frac{i}{2} 98$ & $2136-\frac{i}{2} 100$ & $1915-\frac{i}{2} 94$ & $2036-\frac{i}{2} 98$ & $2014-\frac{i}{2} 97$ \\
\hline \hline
\end{tabular}

\section{E. Influence of the new HEFT constants}

The inclusion of transverse modes in the calculation of the dispersive real part of the amplitude leads unavoidably to consider the case where $M \neq 0$. We have seen how this changes to some extent the location and widths of the amplitudes. On the other hand, the inclusion of the transverse modes leads to the appearance of new counterterms in the HEFT. In the channel $I J=11$ two new low-energy ('anomalous') couplings appear; $a_{3}$ and $\zeta$. Let us see how their presence may affect the previous results.

For this analysis we focus in the four lightest resonances of Table II. This is BP1, BP2, BP1', and BP2' We see from the previous results that, of the two new parameters (not previously considered in unitarization analysis), $a_{3}$ is most relevant as it can be seen in Tables III and IV. Positive values of $a_{3}$ tend to increase the mass of the vector resonance and make it even narrower, making its detection harder. Negative values of $a_{3}$ work in the opposite direction. Although the bounds on $a_{3}$ allow it, the value $\left|a_{3}\right|=0.1$ may be too large, and we also provide $M_{V}$ and $\Gamma_{V}$ for $\left|a_{3}\right|=0.01$. If $a_{3}$ happened to be of the same order as the current bounds for $a_{4}$ and $a_{5}$, its effect would be subleading. The influence of $\zeta$ appears to be less than that of $a_{3}$ but the qualitative behavior remains.

\section{CONCLUSIONS}

One of the main results of this paper is the determination of the one-loop quantum corrections to all the relevant $2 \rightarrow 2$ processes that are relevant to two-Higgs production via the scattering of electroweak gauge bosons in the HEFT. The calculation has been explicitly performed in the 't Hooft-Landau gauge, although physical amplitudes are gauge independent.
In our work, for the first time, a diagrammatic computation of all the on-shell $2 \rightarrow 2$ processes relevant for twoHiggs production is presented. In the one-loop calculation, both transverse and longitudinal polarized modes are included. In the on-shell scheme this necessarily leads to considering the physical values for the Higgs and weak gauge boson masses. ${ }^{5}$ The resulting amplitudes are then unitarized and we analyze the characteristics of the dynamical resonances appearing. An interesting result is that, after unitarization of the partial waves, the effect of including the gauge boson masses is small but significant increasing the mass of the vector resonances typically in the range $2 \%$ to $3 \%$. The widths are unchanged.

The introduction of the transverse degrees of freedom of the gauge bosons also implies the need to consider additional effective couplings that had not been previously considered in unitarization studies. In elastic $W W \rightarrow W W$ scattering, there are two new effective couplings that become relevant. While traditionally the effective couplings $a_{4}$ and $a_{5}$ have been regarded as driving the masses of dynamical resonances, it turns out that the coupling $a_{3}$ (that plays a role only if the a priori subdominant transverse modes are included) is relevant too. It should also be mentioned that while $a_{4}$ and $a_{5}$ are by now fairly constrained by LHC analysis, the bounds on $a_{3}$ are still rather loose. We believe this makes the present study particularly relevant.

The calculation is done on shell, which is what is required for a useful experimental comparison. Keeping redundant operators results in a proliferation of couplings of which only a handful are useful. We also see that the most

\footnotetext{
${ }^{5}$ However, in order to be able to use safely exact isospin relations we work in the custodial limit neglecting electromagnetism, i.e., $g^{\prime}=0$.
} 
influential coefficients in the effective Lagrangian are those surviving the extreme ET limit. There is some logic behind this, but it is reassuring to check it in a detailed calculation.

In the present paper, we have focused on the impact of the new contributions in the vector-isovector channel and have postponed the consideration of the more involved scalar-isoscalar one to a future publication. Unitarization of the latter, that requires a full use of the coupled channel formalism, is most relevant in order to be able to constraint some of the Higgs couplings.

\section{ACKNOWLEDGMENTS}

We thank Oscar Catà and Rafael Delgado for useful discussions. We acknowledge financial support from the State Agency for Research of the Spanish Ministry of Science and Innovation through the "Unit of Excellence María de Maeztu 2020-2023" award to the Institute of Cosmos Sciences (Grant No. CEX2019-000918-M) and from Grants No. PID2019-105614 GB-C21 and No. 2017SGR-929.
[1] G. Aad et al. (ATLAS Collaboration), Phys. Lett. B 716, 1 (2012).

[2] S. Chatrchyan et al. (CMS Collaboration), Phys. Lett. B 716, 30 (2012).

[3] G. F. Giudice, C. Grojean, A. Pomarol, and R. Rattazzi, J. High Energy Phys. 06 (2007) 045.

[4] R. Alonso, M. B. Gavela, L. Merlo, S. Rigolin, and J. Yepes, Phys. Lett. B 722, 330 (2013); 726, 926(E) (2013).

[5] A. Pich, I. Rosell, and J. J. Sanz-Cillero, Phys. Rev. Lett. 110, 181801 (2013).

[6] G. Buchalla, O. Catà, and C. Krause, Nucl. Phys. B880, 552 (2014); B913, 475(E) (2016).

[7] R. Contino, M. Ghezzi, C. Grojean, M. Muhlleitner, and M. Spira, J. High Energy Phys. 07 (2013) 035.

[8] G. Buchalla, O. Cata, A. Celis, and C. Krause, Nucl. Phys. B917, 209 (2017).

[9] A. Pich, arXiv:1804.05664.

[10] D. Espriu and B. Yencho, Phys. Rev. D 87, 055017 (2013).

[11] P. Arnan, D. Espriu, and F. Mescia, Phys. Rev. D 93, 015020 (2016).

[12] A. Pich, I. Rosell, J. Santos, and J. J. Sanz-Cillero, Phys. Rev. D 93, 055041 (2016).

[13] A. Pich, I. Rosell, J. Santos, and J. J. Sanz-Cillero, J. High Energy Phys. 04 (2017) 012.

[14] A. Pich, I. Rosell, and J. J. Sanz-Cillero, Phys. Rev. D 102, 035012 (2020).

[15] D. Espriu, F. Mescia, and B. Yencho, Phys. Rev. D 88, 055002 (2013).

[16] D. Espriu and F. Mescia, Phys. Rev. D 90, 015035 (2014).

[17] R. L. Delgado, A. Dobado, and F. J. Llanes-Estrada, Phys. Rev. D 91, 075017 (2015).

[18] T. Corbett, O. J. P. Éboli, and M. C. Gonzalez-Garcia, Phys. Rev. D 93, 015005 (2016).

[19] R. L. Delgado, A. Dobado, D. Espriu, C. Garcia-Garcia, M. J. Herrero, X. Marcano, and J. J. Sanz-Cillero, J. High Energy Phys. 11 (2017) 098.

[20] C. Garcia-Garcia, M. Herrero, and R. A. Morales, Phys. Rev. D 100, 096003 (2019).

[21] A. Salas-Bernárdez, F. J. Llanes-Estrada, J. EscuderoPedrosa, and J. A. Oller, SciPost Phys. 11, 020 (2021).

[22] A. Dobado and J. R. Pelaez, Phys. Rev. D 65, 077502 (2002).

[23] F. Guerrero and J. A. Oller, Nucl. Phys. B537, 459 (1999).
[24] J. A. Oller, E. Oset, and J. R. Pelaez, Phys. Rev. D 59, 074001 (1999); 60, 099906(E) (1999); 75, 099903(E) (2007).

[25] J. A. Oller, E. Oset, and J. R. Pelaez, Phys. Rev. Lett. 80, 3452 (1998).

[26] A. Dobado and J. R. Pelaez, Phys. Rev. D 56, 3057 (1997).

[27] A. Dobado, M. J. Herrero, and T. N. Truong, Phys. Lett. B 235, 134 (1990).

[28] T. N. Truong, Phys. Rev. Lett. 61, 2526 (1988).

[29] 2020 Update of the European Strategy for Particle Physics (CERN Council, Geneva, 2020), ISBN 978-929083-575-2.

[30] A. Dobado and D. Espriu, Prog. Part. Nucl. Phys. 115, 103813 (2020).

[31] H.-J. He, Y.-P. Kuang, and X.-y. Li, Phys. Lett. B 329, 278 (1994).

[32] C. Grosse-Knetter and I. Kuss, Z. Phys. C 66, 95 (1995).

[33] A. Dobado and J. R. Pelaez, Phys. Lett. B 329, 469 (1994); 335, 554(E) (1994).

[34] A. Dobado and J. R. Peláez, Nucl. Phys. B425, 110 (1994); 434, 475(E) (1995).

[35] M. S. Chanowitz and M. K. Gaillard, Nucl. Phys. B261, 379 (1985).

[36] G. J. Gounaris, R. Kogerler, and H. Neufeld, Phys. Rev. D 34, 3257 (1986).

[37] B. W. Lee, C. Quigg, and H. B. Thacker, Phys. Rev. D 16, 1519 (1977).

[38] J. M. Cornwall, D. N. Levin, and G. Tiktopoulos, Phys. Rev. D 10, 1145 (1974); 11, 972(E) (1975).

[39] G. Buchalla, O. Catà, A. Celis, M. Knecht, and C. Krause, Phys. Rev. D 104, 076005 (2021).

[40] G. Buchalla, O. Cata, A. Celis, M. Knecht, and C. Krause, Nucl. Phys. B928, 93 (2018).

[41] M. J. Herrero and R. A. Morales, Phys. Rev. D 104, 075013 (2021).

[42] M. B. Gavela, K. Kanshin, P. A. N. Machado, and S. Saa, J. High Energy Phys. 03 (2015) 043.

[43] A. Dobado, D. Espriu, and M. J. Herrero, Phys. Lett. B 255, 405 (1991).

[44] G. Altarelli, R. Barbieri, and F. Caravaglios, Nucl. Phys. B405, 3 (1993).

[45] M. E. Peskin and T. Takeuchi, Phys. Rev. D 46, 381 (1992).

[46] R. Alonso, E. E. Jenkins, and A. V. Manohar, J. High Energy Phys. 08 (2016) 101. 
[47] M. Aaboud et al. (ATLAS Collaboration), Phys. Rev. D 95, 032001 (2017).

[48] A. M. Sirunyan et al. (CMS Collaboration), Phys. Lett. B 795, 281 (2019).

[49] A. M. Sirunyan et al. (CMS Collaboration), Phys. Lett. B 798, 134985 (2019).

[50] O. J. P. Eboli, M. C. Gonzalez-Garcia, and J. K. Mizukoshi, Phys. Rev. D 74, 073005 (2006).

[51] O. J. P. Éboli and M. C. Gonzalez-Garcia, Phys. Rev. D 93, 093013 (2016).

[52] M. Rauch, arXiv:1610.08420.

[53] ATLAS Collaboration, Technical Report No. ATL-PHYSPROC-2020-114, CERN, 2019, http://cds.cern.ch/record/ 2693958.

[54] A. M. Sirunyan et al. (CMS Collaboration), J. High Energy Phys. 03 (2021) 257.

[55] M. J. Herrero and E. Ruiz Morales, Nucl. Phys. B418, 431 (1994).

[56] M. Herrero and R. A. Morales, Phys. Rev. D 102, 075040 (2020).

[57] D. Espriu and J. Matias, Phys. Rev. D 52, 6530 (1995).
[58] T. Hahn, Comput. Phys. Commun. 140, 418 (2001).

[59] V. Shtabovenko, R. Mertig, and F. Orellana, Comput. Phys. Commun. 256, 107478 (2020).

[60] V. Shtabovenko, Comput. Phys. Commun. 218, 48 (2017).

[61] G. Passarino and M. J. G. Veltman, Nucl. Phys. B160, 151 (1979).

[62] A. Grozin, in 3rd Dubna International Advanced School of Theoretical Physics (2005) [arXiv:hep-ph/0508242].

[63] R. L. Delgado, A. Dobado, and F. J. Llanes-Estrada, J. High Energy Phys. 02 (2014) 121.

[64] A. Gomez Nicola and J. R. Pelaez, Phys. Rev. D 65, 054009 (2002).

[65] J. de Blas, O. Eberhardt, and C. Krause, J. High Energy Phys. 07 (2018) 048.

[66] G. Aad et al. (ATLAS Collaboration), J. High Energy Phys. 07 (2020) 108.

[67] M. Tanabashi et al. (Particle Data Group), Phys. Rev. D 98, 030001 (2018).

[68] E. da Silva Almeida, A. Alves, N. Rosa Agostinho, O. J. P. Éboli, and M. C. Gonzalez-Garcia, Phys. Rev. D 99, 033001 (2019). 